\title{
Nonlinear Diffusion Limit for a System with Nearest Neighbor Interactions
}

\author{
M. Z. Guo ${ }^{1}$, G. C. Papanicolaou ${ }^{2}$ and S. R. S. Varadhan ${ }^{2 \star}$ \\ ${ }^{1}$ Department of Mathematics, Beijing University, Beijing, People's Republic of China \\ ${ }^{2}$ Courant Institute, New York University, 251 Mercer Street, New York, NY 10012, USA
}

\begin{abstract}
We consider a system of interacting diffusions. The variables are to be thought of as charges at sites indexed by a periodic one-dimensional lattice. The diffusion preserves the total charge and the interaction is of nearest neighbor type. With the appropriate scaling of lattice spacing and time, a nonlinear diffusion equation is derived for the time evolution of the macroscopic charge density.
\end{abstract}

\section{Notation and Summary}

We will study the problem of passage to hydrodynamic limit under diffusion type scaling for a system of charges that are located at various sites of a periodic onedimensional lattice. The charges migrate between adjacent sites randomly according to a well defined diffusion law. The algebraic sum of the charges is always conserved. The charges themselves are of indeterminate sign. Under diffusion type scaling of lattice width and time, a deterministic limit is obtained for the macroscopic charge density and the limit is characterized as the solution of a nonlinear heat equation. We will now develop the notation and end this section with a precise statement of the results as well as a sketch of the proof.

$S$ is the unit circle represented as the interval $0 \leqq \theta \leqq 1$ with end points identified. The scaling parameter is $N$ and the scaled lattice consists of sites $j / N$ in $S$ for $j=1,2, \ldots, N$. The sites adjacent to $j / N$ are $j \pm 1 / N$ with addition being modulo $N$. The charge at site $j / N$ is represented by the variable $x_{j}$. The charge configuration $\left(x_{1}, \ldots, x_{N}\right)$ is represented as a vector $\vec{x}$ in $R^{N}$. The configuration $\vec{x}$ changes with time and as a function of time $\vec{x}(t)$ undergoes a diffusion in $R^{N}$. The infinitesimal generator of the diffusion is given by

$$
L_{N}=\frac{N^{2}}{2} \Sigma\left(\frac{\partial}{\partial x_{i}}-\frac{\partial}{\partial x_{i+1}}\right)^{2}-\frac{N^{2}}{2} \Sigma\left(\phi^{\prime}\left(x_{i}\right)-\phi^{\prime}\left(x_{i+1}\right)\right)\left(\frac{\partial}{\partial x_{i}}-\frac{\partial}{\partial x_{i+1}}\right) .
$$

\footnotetext{
* Work supported by the National Science Foundation under grants no. DMS 8600233 and DMS 8701895
} 
Here $\phi$ is a continuously differentiable function from $R \rightarrow R$ satisfying the properties to be listed below and $\phi^{\prime}(x)=d \phi(x) / d x$. The factor $N^{2}$ in (1.1) with lattice spacing of $1 / N$ represents the effect of diffusion scaling,

$$
\begin{gathered}
\int e^{-\phi(\lambda)} d x=1, \\
\int e^{i x-\phi(x)} d x=M(\lambda)<\infty \quad \forall \lambda \in R,
\end{gathered}
$$

or equivalently there exists $\omega(x)$ which is symmetric and convex on $R$ satisfying

$$
\lim _{x \rightarrow \infty} \frac{|x|}{\omega(x)}=0, \quad \omega(x) \geqq|x| \text { for all } x
$$

and

$$
\int e^{\omega(x)-\phi(x)} d x<\infty
$$

In addition

$$
\int e^{\sigma\left|\phi^{\prime}(x)\right|-\phi(x)} d x<\infty \quad \forall \sigma>0 .
$$

Given such a $\phi$ we can define a density $\Phi_{N}(x)=\exp \left[-\Sigma \phi\left(x_{j}\right)\right]$ on $R^{N}$, and this will be the density relative to Lebesgue measure of a probability measure on $R^{N}$. The generator $L_{N}$ is formally symmetric relative to the density $\Phi_{N}$ and defines a diffusion process with invariant density $\Phi_{N}$ with respect to which the process is reversible. The Dirichlet form for the diffusion is given by

$$
N^{2} \hat{D}_{N}(u)=\frac{N^{2}}{2} \int\left\{\Sigma\left(\frac{\partial u}{\partial x_{i}}-\frac{\hat{c} u}{\partial x_{i+1}}\right)^{2}\right\} \Phi_{N} d \vec{x} .
$$

The diffusion is not ergodic for the invariant measure $\Phi_{N}$. The sum $x_{1}+\cdots+x_{N}$ is conserved by the diffusion and the hyperplanes $x_{1}+\cdots+x_{N}=N y$ of average charge $y$ are invariant sets. For every $y$ the diffusion restricted to such a hyperplane is elliptic and ergodic. The invariant measure on the hyperplane is the conditional distribution of $\Phi_{N}$ given $x_{1}+\cdots+x_{N} / N=y$.

We start with an initial distribution for $\vec{x}(0)$ given by some density $f_{N}^{0}\left(x_{1}, \ldots, x_{N}\right)$ relative to $\Phi_{N}$. Then the evolution will give us a density $f_{N}^{t}\left(x_{1}, \ldots, x_{N}\right)$ for $t \geqq 0$. To obtain $f_{N}^{t}$ we have to solve the heat equation

$$
\frac{\partial f_{N}^{t}}{\partial t}=L_{N} f_{N}^{t} \text { for } t>0,\left.\quad f_{N}^{t}\right|_{t=0}=f_{N}^{0} .
$$

Associated with the charge configuration $\vec{x}$ we have the measure,

$$
\mu_{N}=\frac{1}{N} \Sigma x_{j} \delta_{j / N}
$$

viewed as a random signed measure on $S$. If for every smooth function $J(\theta)$ on $S$,

$$
\lim _{N \rightarrow \infty}\left\langle J, \mu_{N}\right\rangle=\lim _{N \rightarrow \infty} \frac{1}{N} \Sigma J\left(\frac{i}{N}\right) x_{i}=\int J(\theta) m(\theta) d \theta
$$

exists, then we say that $m$ is the asymptotic macroscopic charge density. Of our initial density $f_{N}^{0}$ we assume that for some nice $m_{0}(\theta)$, every smooth $J(\cdot)$ and 
each $\delta>0$,

$$
\lim _{N \rightarrow \infty} \int_{E_{N}} f_{N}^{0} \Phi_{N} d x=0
$$

where

$$
E_{N}=\left[\vec{x}:\left|\frac{1}{N} \Sigma J\left(\frac{i}{N}\right) x_{i}-\int m_{0}(\theta) J(\theta) d \theta\right| \geqq \delta\right] .
$$

In addition we assume that the entropy

$$
H_{N}\left(f_{N}^{0}\right)=\int f_{N}^{0} \log f_{N}^{0} \Phi_{N} d \vec{x}
$$

satisfies a bound

$$
H_{N}\left(f_{N}^{0}\right) \leqq C N
$$

for some constant $C$.

Under these conditions the randomly changing charge configurations at time $t \geqq 0$ are shown to have an asymptotic deterministic charge density $m(t, \theta)$ which is characterized as the unique solution of a certain nonlinear heat equation with initial condition $m_{0}(\theta)$. But to describe this equation we have to develop some more notation. We look at the function $M(\hat{\lambda})$ defined in (1.3) and define

$$
\rho(\lambda)=\log M(\lambda), \quad h(y)=\sup _{\lambda}[\lambda y-\rho(\lambda)] .
$$

Then $h(\cdot)$ and $\rho(\cdot)$ are a pair of conjugate convex functions and

$$
\lambda=h^{\prime}(y) \text { if and only if } y=\rho^{\prime}(\lambda) \text {. }
$$

i.e. $h^{\prime}$ and $\rho^{\prime}$ are inverses of each other. By elementary calculation one can check that

$$
\frac{1}{M(\lambda)} \int e^{i x-\phi(x)} x d x=\rho^{\prime}(\hat{\lambda})
$$

so that the value of $\lambda$ that makes (1.14) equal to $y$ is $\lambda=h^{\prime}(y)$. One knows also that $\rho^{\prime}$ and $h^{\prime}$ are smooth strictly increasing functions. We can now state our main result.

Theorem 1.1. If the initial distribution of charges satisfies (1.9) and (1.11), then for every $t>0$, arbitrary smooth $J(\cdot)$ and each $\delta>0$,

with

$$
\lim _{N \rightarrow \infty} \int_{E_{N}^{t}} f_{N}^{t} \Phi_{N} d \vec{x}=0
$$

$$
E_{N}^{t}=\left\{\left(x_{1}, \ldots, x_{N}\right):\left|\frac{1}{N} \Sigma J\left(\frac{1}{N}\right) x_{i}-\int m(\theta, t) J(\theta) d \theta\right| \geqq \delta\right\},
$$

where $m(\theta, t)$ is the unique weak solution of the nonlinear diffusion equation

$$
\frac{\partial m}{\partial t}=\frac{1}{2}\left[h^{\prime}(m(\theta, t))\right]_{\theta \theta},\left.\quad m(t, \theta)\right|_{t=0}=m_{0}(\theta) .
$$

We will now give a sketch of the proof. 
Let us fix a smooth $J$ and consider the stochastic process

$$
\xi_{N}(t)=\frac{1}{N} \Sigma J\left(\frac{i}{N}\right) x_{i}(t)
$$

It is easy to write down a stochastic differential equation satisfied by $\breve{\zeta}_{N}(t)$ in the form

$$
d \xi_{N}(t)=a_{N}(t) d \beta+b_{N}(t) d t
$$

The way the problem is scaled implies that $a_{N}(t) \rightarrow 0$ as $N \rightarrow \infty$. An explicit calculation of $b_{N}(t)$ can be made and we obtain

$$
b_{N}(t)=\frac{N^{2}}{2} \Sigma J\left(\frac{i}{N}\right)\left[\phi^{\prime}\left(x_{i-1}(t)\right)-2 \phi^{\prime}\left(x_{i}(t)\right)+\phi^{\prime}\left(x_{i+1}(t)\right)\right] .
$$

Using summation by parts and the smoothness of $J(\theta)$ we can pass to

$$
b_{N}(t) \simeq \frac{1}{2} \Sigma J^{\prime \prime}\left(\frac{i}{N}\right) \phi^{\prime}\left(x_{i}(t)\right) .
$$

At this point one has to justify the ansatz that the density $f_{N}^{t}\left(x_{1}, \ldots, x_{N}\right)$ looks like a slowly varying family of local Gibbs states. This means that averages of $\phi^{\prime}\left(x_{i}\right)$ can be replaced by their mean values $(1 / M(\lambda)) \int e^{\lambda-\phi(x)} \phi^{\prime}(x) d x$, where $\lambda=h^{\prime}(m)$ and $m$ is the local macroscopic density. One calculates easily that

$$
\frac{1}{M(\lambda)} \int e^{\prime x-\phi(x)} \phi^{\prime}(x) d x=\lambda=h^{\prime}(m)
$$

and therefore

$$
\lim _{N \rightarrow \infty} b_{N}(t)=\frac{1}{2} \int J^{\prime \prime}(\theta) h^{\prime}(m(\theta, t)) d \theta .
$$

The crucial step in the whole proof is the replacement of (1.19) by (1.21). Since the ansatz is too vague to be formulated rigorously we obtain certain elementary bounds on the average $1 / T \int_{0}^{T} f_{N}^{t}\left(x_{1}, \ldots, x_{N}\right) d t$ as a consequence of (1.11). These are then shown to imply certain inequalities, which in turn justify the passage from (1.19) to (1.21).

\section{Entropy and its Rate of Change}

Since we will have to consider probability measures on $R^{N}$ as well as many of its projections we now introduce some uniform notation. A probability measure $\alpha$ on $R^{N}$, if it is absolutely continuous will be represented by its density $f$ with respect to the density $\Phi_{N}$ so that its actual Lebesgue density is $f \Phi_{N}$. If $F \subset\{1,2, \ldots, N\}$ is a subset of cardinality $k$ we can project $\alpha$ from $R^{N}$ to $R^{k}$ by looking at the distribution of $\left\{x_{j}\right\}$ for $j \in F$. We will denote the projection by $\alpha^{F}$ and the corresponding density on $R^{k}$, relative to $\Phi_{k}$ by $f^{F}$. We have the obvious formula

$$
f^{F}\left(x_{F}\right)=\int f(x) \Phi_{F^{c}}\left(x_{F^{c}}\right) d x_{F^{c}} .
$$


We have used the obvious notation

$$
\Phi_{A}(x)=\exp \left[-\sum_{j \in A} \phi\left(x_{j}\right)\right], \quad x_{A}=\left\{x_{j}: j \in A\right\}, \quad d x_{A}=\prod_{j \in A} d x_{j} .
$$

We consider the following diffusion generators on $R^{N}$ defined for a pair $(i, j) \in$ $[1,2, \ldots, N]$,

$$
\hat{L}_{i, j}=\frac{1}{2}\left(\frac{\partial}{\partial x_{i}}-\frac{\partial}{\partial x_{j}}\right)^{2}-\frac{1}{2}\left(\phi^{\prime}\left(x_{i}\right)-\phi^{\prime}\left(x_{j}\right)\right)\left(\frac{\partial}{\partial x_{i}}-\frac{\partial}{\partial x_{j}}\right) .
$$

$\hat{L}_{i, j}$ can be viewed as an operator acting on functions depending on the variables $x_{F}=\left\{x_{j}: j \in F\right\}$, provided $i, j \in F$. One should think of $\hat{L}_{i, j}$ as representing the exchange of charge between sites $i$ and $j$ along a "direct bond" linking them. Our generator

$$
\underline{L}_{N}=N^{2} \sum_{i=0}^{N-1} \hat{L}_{i, i+1}
$$

is a sum over nearest neighbor bonds. But we can consider sums that involve other bonds as well. Each $\hat{L}_{i, j}$ is reversible relative to the weight $\Phi_{N}$.

There are two functionals on probability measures on $R^{N}$ that are relevant for our purpose. The first one is the entropy. If $\alpha$ is a probability distribution with density $f$ then

$$
H_{N}(\alpha)=\int_{R^{N}} f \log f \Phi_{N} d \vec{x}
$$

It is known that entropy is a lower semicontinuous convex functional of $\alpha$ and can be defined equivalently by the basic entropy inequality as the smallest constant $H$ for which

$$
\int U f \Phi_{N} d x \leqq \log \int e^{U} \Phi_{N} d x+H
$$

for all bounded measurable functions $U$ on $R^{N}$.

If we let the initial distribution evolve according to the forward equation or equivalently the density $f$ evolve according to (1.8), then the function

$$
H_{N}(t)=H\left(\alpha_{N}^{t}\right)=\int f_{N}^{t} \log f_{N}^{t} \Phi_{N} d x
$$

is nonincreasing in $t$ and one can compute

$$
\frac{d H_{N}(t)}{d t}=-\frac{N^{2}}{2} \int\left\{\sum_{i=0}^{N-1} \frac{1}{f_{N}^{t}}\left(\frac{\partial f_{N}^{t}}{\partial x_{i}}-\frac{\partial f_{N}^{t}}{\partial x_{i+1}}\right)^{2}\right\} \Phi_{N} d x .
$$

Corresponding to each operator $\hat{L}_{i, j}$ we can define the Dirichlet form

$$
\hat{D}_{i, j}(u)=\frac{1}{2} \int_{R^{N}}\left(\frac{\partial u}{\partial x_{i}}-\frac{\partial u}{\partial x_{j}}\right)^{2} \Phi_{N} d x
$$

and the corresponding $\hat{I}_{i, j}(f)$ by

$$
\hat{I}_{i, j}(f)=\hat{D}_{i, j}(\sqrt{f})=\frac{1}{8} \int \frac{1}{f}\left(\frac{\partial f}{\partial x_{i}}-\frac{\partial f}{\partial x_{j}}\right)^{2} \Phi_{N} d x
$$


We can rewrite (2.4) in the form

$$
\frac{d H_{N}(t)}{d t}=-4 N^{2} \sum_{j=0}^{N-1} \hat{I}_{j, j+1}\left(f_{N}^{t}\right)
$$

The variational characterization (2.3) of entropy defines $H_{N}(\alpha)$ as a natural lower semicontinuous convex functional of $\alpha$ which is finite only when $\alpha$ has a density $f$ for which $\int f \log f \Phi_{N} d x$ is finite. Similarly there is also a variational characterization of $I(\alpha)$,

$$
I(\alpha)=\sup _{u>0}\left[-\int\left(\frac{L u}{u}\right)(x) \alpha(d x)\right] .
$$

If $\alpha$ should have a nice density $f$ then

$$
I(\alpha)=D(\sqrt{f})
$$

where $L$ is any self adjoint operator with respect to $\Phi_{N}$ and $D$ is the Dirichlet form of $L$. Typically $L=L_{N}$ or $\underline{L}_{i, j}$. Since $L$ is not elliptic $\hat{I}(\alpha)$ can be finite without $\alpha$ having a density $f$. However the restriction of $\alpha$ to any invariant hyperplane will have a density relative to the restriction of $\Phi_{N}$ to the hyperplane and the Dirichlet form is only computed from tangential derivatives on the hyperplane.

We want to establish some simple inequalities relating to the behavior of $I(f)$ under projections. Let $\alpha$ be a probability measure on $R^{N}$ and $\alpha^{F}$ its projection onto $R^{k}$ corresponding to $\left\{x_{j}: j \in F\right\}$, where $k$ is the cardinality of $F$. We then have

Lemma 2.1. For any pair $i, j \in F$,

$$
\hat{I}_{i, j}\left(\alpha^{F}\right) \leqq \hat{I}_{i, j}(\alpha)
$$

Proof.

$$
\hat{I}_{i, j}\left(\alpha^{F}\right)=\sup _{u>0}\left[-\int\left(\frac{L_{i j} u}{u}\right)\left(x_{F}\right) \alpha^{F}\left(d x_{F}\right)\right]
$$

over all functions $u$ of $x_{F}$. Since $\alpha^{F}$ is the projection of $\alpha$,

$$
\hat{I}_{i, j}\left(\alpha^{F}\right) \leqq \sup _{u>0}\left[-\int\left(\frac{L_{i j} u}{u}\right)(x) \alpha(d x)\right]=\hat{I}_{i, j}(\alpha) .
$$

The supremum in (2.11) is only over functions of $x_{F}$, and one gets the inequality in $(2.12)$ because the supremum there is over a larger class, namely all functions of $x$.

Let $f$ be a probability density on $R^{N}$. Let $l \geqq 1$ be given. We consider the set $F_{i, l}=\{j:|j-i| \leqq l\}$ and $f^{i, l}=f^{F_{i, l}}$ the projection of $f$ onto $R^{2 l+1}$. We define $f^{l}=(1 / N) \sum_{i=1}^{N} f^{i, l}$ as a measure on $R^{2 l+1}$.

Lemma 2.2. For any $l \geqq 1$ and $\alpha$,

$$
\sum_{i=-l}^{l-1} \hat{I}_{i, i+1}\left(f^{l}\right) \leqq \frac{2 l}{N} \sum_{i=0}^{N-1} \hat{I}_{i, i+1}(f) .
$$


Remark. We have abused the notation somewhat here. On the left $f^{l}$ is a measure on $R^{2 l+1}$ with coordinates indexed by variables $\left\{x_{j}:|j| \leqq l\right\}$ and $\hat{I}_{i, i+1}$ are for nearest neighbor bonds between them. But the sites $|j| \leqq l$ are arranged in a chain with two ends. On the right however the $N$ sites are arranged cyclically.

Proof. By convexity

$$
\begin{aligned}
\hat{I}_{i, i+1}\left(f^{l}\right) & =\hat{I}_{i, i+1}\left(\frac{1}{N} \sum_{r=1}^{N} f^{r, l}\right) \leqq \frac{1}{N} \sum_{r=1}^{N} \hat{I}_{i, i+1}\left(f^{r, l}\right) \\
& =\frac{1}{N} \sum_{r=1}^{N} \hat{I}_{r+i, r+i+1}\left(f^{r, l}\right) \quad \text { [by abuse of notation] } \\
& \leqq \frac{1}{N} \sum_{r=1}^{N} \hat{I}_{r+i, r+i+1}(f) \quad \text { [by Lemma 2.1] } \\
& =\frac{1}{N} \sum_{r=1}^{N} \hat{I}_{r, r+1}(f) .
\end{aligned}
$$

This proves the lemma.

Similarly let $f$ on $R^{N}$ be given along with $l \geqq 1$ and $m$ such that $2 l+1 \leqq m \leqq$ $N-(2 l+1)$. Consider the set $F_{m, l, i}=\{j:|j-i| \leqq l$ or $|j-m-i| \leqq l\}$. We project $f$ onto the coordinates in $F_{m, l, i}$ and the resulting density on $R^{2 l+1} \times R^{2 l+1}$ is denoted by $f^{m, l, i}$. We average this over location $i$ and denote $f^{m, l}=(1 / N) \sum_{i=1}^{N} f^{m, l, i}$. For clarity we think of the variables in $R^{2 l+1} \times R^{2 l+1}$ as $\left\{y_{j}:|j| \leqq l\right\}$ and $\left\{z_{j}:|j| \leqq l\right\}$. We define three forms

$$
\begin{aligned}
\hat{I}_{i, i+1}^{Y}(f) & =\frac{1}{8} \int \frac{1}{f}\left(\frac{\partial f}{\partial y_{i}}-\frac{\partial f}{\partial y_{i+1}}\right)^{2} \Phi_{2 l+1}(y) \Phi_{2 l+1}(z) d y d z \\
\hat{I}_{i, i+1}^{Z}(f) & =\frac{1}{8} \int \frac{1}{f}\left(\frac{\partial f}{\partial z_{i}}-\frac{\partial f}{\partial z_{i+1}}\right)^{2} \Phi_{2 l+1}(y) \Phi_{2 l+1}(z) d y d z \\
\hat{I}^{Y, Z}(f) & =\frac{1}{8} \int \frac{1}{f}\left(\frac{\partial f}{\partial z_{0}}-\frac{\partial f}{\partial y_{0}}\right)^{2} \Phi_{2 l+1}(y) \Phi_{2 l+1}(z) d y d z
\end{aligned}
$$

We then have the analog of Lemma 2.2.

Lemma 2.3. For any $m$ such that $2 l+1 \leqq m \leqq N-(2 l+1)$,

$$
\begin{aligned}
\sum_{i=-l}^{l-1} \hat{I}_{i, i+1}^{Y}\left(f^{m, l}\right) & \leqq \frac{2 l}{N} \sum_{i=0}^{N-1} \hat{I}_{i, i+1}(f), \\
\sum_{i=-l}^{l-1} \hat{I}_{i, i+1}^{Z}\left(f^{m, l}\right) & \leqq \frac{2 l}{N} \sum_{i=0}^{N-1} \hat{I}_{i, i+1}(f), \\
I^{Y, Z}\left(f^{m, l}\right) & \leqq \frac{m^{2}}{N} \sum_{i=0}^{N-1} \hat{I}_{i, i+1}(f) .
\end{aligned}
$$

Proof. Inequalities (2.12) and (2.15) are analogs of inequality of (2.13). We therefore 
only have to prove (2.16). By convexity and (2.10),

$$
\hat{I}^{Y, Z}\left(f^{m, l}\right) \leqq \frac{1}{N} \sum_{i=0}^{N-1} \hat{I}^{Y, Z}\left(f^{m, l, i}\right) \leqq \frac{1}{N} \sum_{i=0}^{N-1} \hat{I}_{i, i+m}(f)
$$

By Schwartz's inequality,

$$
\hat{I}_{i, i+l}(f) \leqq l \sum_{j=i}^{i+l-1} \hat{I}_{j, j+1}(f) .
$$

Summing with respect to $i$ from $i=0$ to $N-1$ we get (2.16).

Let us start our evolution with an initial distribution satisfying (1.1). Then according to formula (2.4) we obtain the following estimates:

and

$$
\int f_{N}^{t} \log f_{N}^{t} \Phi_{N} d x \leqq C N \text { for all } t \geqq 0,
$$

$$
\frac{1}{2} \int_{0}^{T} d t\left\{\left\{\sum_{i=0}^{N-1} \frac{1}{f_{N}^{t}}\left(\frac{\partial f_{N}^{t}}{\partial x_{i}}-\frac{\partial f_{N}^{t}}{\partial x_{i+1}}\right)^{2}\right\} \Phi_{N} d x \leqq \frac{C}{N} \text { for all } T .\right.
$$

Now if we use the fact that both the expressions $\int f \log f \Phi_{N} d x$ and

$$
\frac{1}{2} \int\left\{\sum_{i=0}^{N-1} \frac{1}{f}\left(\frac{\partial f}{\partial x_{i}}-\frac{\partial f}{\partial x_{i+1}}\right)^{2}\right\} \Phi_{N} d x
$$

are convex functionals of $f$, we obtain for the density $(1 / T) \int_{0}^{T} f_{N}^{t} d t$ or taking $T=1$ and setting

\section{Theorem 2.4.}

$$
\bar{f}_{N}=\int_{0}^{1} f_{N}^{t} d t
$$

$$
\begin{gathered}
\int \bar{f}_{N} \log \bar{f}_{N} \Phi_{N} d x \leqq C N \\
\frac{1}{2}\left\{\left\{\sum_{i=1}^{N-1} \frac{1}{\bar{f}_{N}}\left(\frac{\partial \bar{f}_{N}}{\partial x_{i}}-\frac{\partial \bar{f}_{N}}{\partial x_{i+1}}\right)^{2}\right\} \Phi_{N} d x \leqq \frac{C}{N} .\right.
\end{gathered}
$$

In addition

$$
\sup _{N}\left\{\frac{1}{N} \sum \omega\left(x_{i}\right)\right\} \bar{f}_{N} \Phi_{N} d x \leqq C^{\prime} .
$$

Proof. Only (2.21) needs an explanation. By estimate (2.3) and (2.19),

$$
\int\left\{\sum \omega\left(x_{i}\right)\right\} \bar{f}_{N} \phi_{N} d x \leqq \log \int e^{\Sigma \omega\left(x_{i}\right)} \Phi_{N} d x+C N \leqq C_{1} N+C N .
$$

\section{Limit Theorems for Densities}

The aim of this section is to prove two theorems concerning the probability densities of sums of independent random variables. Although the results are essentially well known we need special forms of these results for our use. We will give a quick sketch of the proofs as we go along.

Lemma 3.1. Let $f(x)$ be a probability density on $R$ such that $\int x f(x) d x=0$, 
$\int x^{2} f(x) d x=\sigma^{2}, \int x^{4} f(x) d x \leqq M$ and $\int\left|f^{\prime}(x)\right| d x \leqq M$. Then there is a number $\delta=\delta(M)$ depending only on $M$ such that

$$
\left|\int e^{i \xi x} f(x) d x\right| \leqq\left(1+|\xi|^{2}\right)^{-\delta} \text { for all } \xi \in R \text {. }
$$

Proof. If $\phi(\xi)=\int e^{i \xi x} f(x) d x$, then $|\phi(\xi)| \leqq 1$ and moreover

$$
\begin{aligned}
\left|\phi(\xi)-1+\frac{\sigma^{2} \xi^{2}}{2}\right| \leqq C_{M}|\xi|^{4}, \\
|\phi(\xi)| \leqq \frac{C_{M}}{|\xi|},
\end{aligned}
$$

and for every $\varepsilon>0$,

$$
\sup _{|\xi| \geqq \varepsilon}|\phi(\xi)| \leqq \theta_{M}(\varepsilon)<1,
$$

where $C_{M}$ and $\theta_{M}(\varepsilon)$ are constants depending only on $M$ and $\varepsilon>0$ as indicated. Moreover $\sigma^{2}$ is bounded above as well as below away from zero in terms of $M$. To establish (3.1) for all $\xi$ we use (3.2) for small $|\xi|$, (3.3) for large $|\xi|$ and (3.4) for intermediate $|\xi|$.

Lemma 3.2. Let $f_{n}(x)$ be the density of $x_{1}+\cdots+x_{n} / \sqrt{n}$, where $x_{1}, \ldots, x_{n}$ are independent and identically distributed with a common density $f(x)$ satisfying the assumptions of Lemma 3.1. Then

$$
\lim _{n \rightarrow \infty} f_{n}(x)=\frac{1}{\sqrt{2 \pi \sigma}} e^{-x^{2} / 2 \sigma^{2}}
$$

uniformly on $R$ and in fact the derivatives of $f_{n}(x)$ of all orders converge uniformly to the corresponding derivatives.

Proof. We use the formula

$$
f_{n}(x)=\frac{1}{2 \pi} \int e^{-i x \xi}\left[\phi\left(\frac{\xi}{\sqrt{ } n}\right)\right]^{n} d \xi .
$$

For sufficiently large $n$, the above formula is well defined because of inequality (3.1). Clearly $[\phi(\xi / \sqrt{ } n)]^{n} \rightarrow e^{-\left(\xi^{2} / 2\right) \sigma^{2}}$ as $n \rightarrow \infty$. It is then a question of verifying that we can apply the dominated convergence theorem. From the monotonicity of the function $(1+A / t)^{t}$ in $t$ for $t \geqq 0$ we can estimate

$$
\sup _{n \geqq l}|\xi|^{k}\left|\phi\left(\frac{\xi}{\sqrt{ } n}\right)\right|^{n} \leqq \sup _{n \geqq l}|\xi|^{k}\left(1+\frac{|\xi|^{2}}{n}\right)^{-n \delta} \leqq|\xi|^{k}\left(1+\frac{|\xi|^{2}}{l}\right)^{-l \delta},
$$

which is integrable for $l$ large enough. We can now differentiate (3.6) with respect to $x$ as often as we want and pass to the limit.

Let $e^{-\phi(x)}$ be our basic probability density and $M(\lambda)=\int e^{\lambda x-\phi(x)} d x$. We denote $\rho(\hat{\lambda})=\log M(\lambda)$ and

$$
\rho^{\prime}(\lambda)=\frac{M^{\prime}(\lambda)}{M(\lambda)}=\int \frac{1}{M(\lambda)} e^{\lambda x-\phi(x)} x d x
$$


Let us define

Then

$$
f(x, \lambda)=\frac{1}{M(\lambda)} e^{\lambda\left(x+\rho^{\prime}(\lambda)\right)} e^{-\phi\left(x+\rho^{\prime}(\lambda)\right)}
$$

and

$$
\begin{gathered}
\int f(x, \lambda) d x=1, \\
\int x f(x, \lambda) d x=0, \\
\int x^{2} f(x, \lambda) d x=\sigma^{2}(\lambda)=\frac{M^{\prime \prime}(\lambda)}{M(\lambda)}-\left[\frac{M^{\prime}(\lambda)}{M(\lambda)}\right]^{2},
\end{gathered}
$$

is bounded if $\lambda$ is bounded.

Lemma 3.3. Let $f_{n}(x, \lambda)$ be the probability density of $x_{1}+\cdots+x_{n} / \sqrt{ } n$, where $x_{1}, \ldots, x_{n}$ are independent and identically distributed having a common density $f(x, \lambda)$. Then

$$
\lim _{n \rightarrow \infty} f_{n}(x, \lambda)=\frac{1}{\sqrt{2 \pi} \sigma(\lambda)} \exp \left[-\frac{1}{2 \sigma^{2}(\lambda)} x^{2}\right]
$$

uniformly in $x$ and bounded $\lambda$ intervals. In addition the first partial derivative of $f_{n}$ with respect to $\lambda$ converges to the corresponding partial derivative on the right uniformly on bounded $\lambda$ sets and uniformly in $x$.

Proof. Let

Then

$$
\psi(\xi, \hat{\lambda})=\int e^{i \xi x} f(x, \hat{\lambda}) d x
$$

$$
f_{n}(x, \lambda)=\frac{1}{2 \pi} \int e^{-i \xi x}\left[\psi\left(\frac{\xi}{\sqrt{n}}, \lambda\right)\right]^{n} d \xi .
$$

Let us compute $\partial f_{n} / \partial \lambda$. Computing the derivative inside we note first

$$
\frac{\partial \psi}{\partial \lambda}=\int e^{i \xi x} \frac{\partial f}{\partial \lambda}(x, \lambda) d x=\int\left(e^{i \xi x}-1-i \xi x\right) \frac{\partial f}{\partial \lambda}(x, \lambda) d x,
$$

because $\int(\partial f / \partial \lambda)(x, \lambda) d x=\int x(\partial f / \partial \lambda)(x, \lambda) d x=0$. Therefore

Therefore

$$
\left|\frac{\partial \phi}{\partial \lambda}\right| \leqq|\xi|^{2} \int x^{2}\left|\frac{\partial f}{\partial \lambda}(x, \lambda)\right| d x \leqq C_{\lambda}|\xi|^{2} .
$$

$$
\left|n \frac{\partial \phi}{\partial \lambda}\left(\frac{\xi}{\sqrt{n}}, \lambda\right)\right| \leqq n \cdot C_{\lambda}\left(\frac{|\xi|}{\sqrt{n}}\right)^{2} \leqq C_{\lambda}|\xi|^{2} .
$$

We are now ready to prove our first main theorem of the section:

Theorem 3.4. Let $\phi_{n}(x)$ be the density of $x_{1}+\cdots+x_{n} / n$, where $x_{1}, \ldots, x_{n}$ are independent and identically distributed with a common density $e^{-\phi(x)}$. Then 


$$
\begin{aligned}
\lim _{n \rightarrow \infty} \frac{1}{n} \log \phi_{n}(x) & =-h(x), \\
\lim _{n \rightarrow \infty} \frac{1}{n} \frac{\phi_{n}^{\prime}(x)}{\phi_{n}(x)} & =-h^{\prime}(x),
\end{aligned}
$$

uniformly on compact $x$-intervals.

Proof. We use Cramer's trick. Let $y \in R, \lambda=h^{\prime}(y)$ and $y=\rho^{\prime}(\lambda)$. Consider

$$
f(x, y)=\frac{1}{M(\lambda)} e^{j(x+y)-\phi(x+v)} .
$$

We want to apply Lemma 3.3 to the above density treating $y$ as a parameter. Then density $f_{n}(x, y)$ of $x_{1}+\cdots+x_{n} / \sqrt{n}$ at $x=0$ is related to the density $\hat{f}_{n}(x, y)$ of $x_{1}+\cdots+x_{n} / \sqrt{n}$ at $x=0$ by a simple factor of $\sqrt{n}$ and a calculation yields

$$
\phi_{n}(y)=[M(\lambda)]^{n} e^{-n \lambda y} \sqrt{n} f_{n}(0, y) .
$$

We also know that $\log M(\lambda)-\lambda y=\rho(\lambda)-\lambda y=-h(y)$. Equations (3.14) and (3.15) are now easy consequences of Lemma 3.3.

We are now interested in studying the conditional distribution of $x_{1}, \ldots, x_{n}$ on the hyperplane $x_{1}+\cdots+x_{n} / n=y$, where $x_{1}, \ldots, x_{n}$ are independent and identically distributed random variables with a common density $e^{\phi(x)}$. We have clearly a smooth density $\phi_{n}(x)$ for $x_{1}+\cdots+x_{n} / n$, and therefore the conditional density is well defined on each hyperplane. Let us denote by $v_{y}^{(n)}\left(d x_{1}, \ldots, d x_{n}\right)$ the measure on $R^{n}$ concentrated on the hyperplane $x_{1}+\cdots+x_{n} / n=y$. We want to establish the following main theorem.

Theorem 3.5. Let $F$ be a bounded continuous function on $R^{k}$ for some $k \geqq 1$. Let for each $y \in R, \alpha_{y}(d x)$ denote the probability measure on $R$ with density $(1 / M(\lambda)) e^{i x-\phi(x)}$, where $\lambda=h^{\prime}(y)$. Then for every $\varepsilon>0$,

$$
\begin{array}{r}
\lim _{n \rightarrow \infty} v_{y}^{(n)}\left[\left(x_{1}, \ldots, x_{n}\right): \mid \frac{1}{n-k+1} \sum_{i=1}^{n-k+1} F\left(x_{i}, \ldots, x_{i+k-1}\right)\right. \\
\left.\quad-\int F\left(z_{1}, \ldots, z_{k}\right) \alpha_{y}\left(d z_{1}\right) \alpha_{y}\left(d z_{2}\right) \cdots \alpha_{y}\left(d z_{k}\right) d y \mid \geqq \varepsilon\right]=0
\end{array}
$$

locally uniformly in $y \in R$.

Proof. Let us remark that $v_{y}^{(n)}\left(d x_{1}, \ldots, d x_{n}\right)$ is symmetric with respect to the permutation group acting on the variables $x_{1}, \ldots, x_{n}$. Therefore by Hewitt-Savage 0-1 law and its implication we need to check the theorem only when $k=1$ and $F(x)$ is a bounded continuous function on $R$ with a uniformly bounded first derivative. This will follow from the following lemma.

Lemma 3.6. For every $\theta \in R$

$$
\lim _{n \rightarrow \infty} \frac{1}{n} \log \int e^{\theta\left(F\left(x_{1}\right)+\cdots+F\left(x_{n}\right)\right)} d v_{y}^{(n)}\left(d x_{1}, \ldots, d x_{n}\right)=G(\theta, y)
$$


exists and the limit $G(\theta)$ is differentiable at $\theta=0$ with

$$
\left.\frac{d G(\theta, y)}{d \theta}\right|_{\theta=0}=\int F(x) \alpha_{y}(d x) .
$$

Proof. Let us denote by $H_{n}(y)$ the function

$$
\begin{aligned}
H_{n}(y, \theta) & =\int_{x_{1}+\cdots+x_{n}=n y} e^{\theta\left(F\left(x_{1}\right)+\cdots+F\left(x_{n}\right)\right)-\phi\left(x_{1}\right)-\phi\left(x_{2}\right)--\phi\left(x_{n}\right)} d x_{1} \cdots d x_{n}, \\
\phi_{n}(y) & =\int_{x_{1}+\cdots+x_{n}=n y} e^{-\sum \phi\left(x_{j}\right)} d x_{1} \cdots d x_{n} .
\end{aligned}
$$

Proving (3.18) reduces to showing that

$$
\lim _{n \rightarrow \infty} \frac{1}{n} \log H_{n}(y, \theta)-\lim _{n \rightarrow \infty} \frac{1}{n} \log \phi_{n}(y)=G(\theta, y)
$$

exists locally uniformly in $y$. Let us denote

$$
a(\theta)=\int e^{\theta F(x)-\phi(x)} d x, \quad M(\lambda, \theta)=\frac{1}{a(\theta)} \int e^{x+\theta F(x)-\phi(x)} d x .
$$

Then essentially by replacing $\phi(x)$ with $\phi(x)-\theta F(x)$ we can apply Theorem 3.4 and obtain

where

$$
\lim _{n \rightarrow \infty} \frac{1}{n} \log H_{n}(y, \theta)=\log a(\theta)-h(\theta, y)
$$

$$
h(\theta, y)=\sup _{\lambda}[\lambda y-\log M(\lambda, \theta)] .
$$

Equation (3.18) now follows with

$$
G(\theta, y)=\log a(\theta)-h(\theta, y)+h(y) .
$$

It is now elementary to conclude that

$$
\left.\frac{d G(\theta, y)}{d \theta}\right|_{\theta=0}=\frac{a^{\prime}(0)}{a(0)}+\left.\frac{1}{M\left(\lambda^{*}, 0\right)} \frac{\partial M}{\partial \theta}\right|_{\left(\lambda^{*}, 0\right)},
$$

where $\lambda^{*}=h^{\prime}(y)$. The right-hand side of (3.20) is easily computed to be $(1 / M(\lambda)) \int F(x) e^{i^{*} \lambda-\phi(\lambda)} d x$. This proves (3.19).

Theorem 3.5 follows from Lemma 3.6 by exponential Tchebychev bounds.

\section{Local Gibbs States}

A local Gibbs state is a vague term which refers to a probability distribution on $R^{N}$ with a density relative to $\Phi_{N}$ which looks somewhat like $c \exp \left[\Sigma \lambda_{i} x_{i}\right]$. Here $\lambda_{i}$ are constants that are slowly varying so that $\lambda_{i}=\bar{\lambda}(i / N)$ for some smooth function $\bar{\lambda}$ on $S$. If we define $\bar{m}(\theta)=\rho^{\prime}(\bar{\lambda}(\theta))$, then the above local Gibbs state corresponds to a macroscopic charge density of $\bar{m}(\theta)$ on $S$. This refers to the fact that if $J(\theta)$ is a smooth function on $S$ then $(1 / N) \Sigma J(i / N) x_{i}$ as a random variable on $R^{N}$ is almost a constant for large $N$, being nearly equal to $\int J(\theta) \bar{m}(\theta) d \theta$ under our local 
Gibbs density. For such a density $f=c \exp \left[\Sigma \lambda_{i} x_{i}\right]$ one can calculate

$$
\begin{aligned}
\sum_{i=1}^{N} \hat{I}_{i, i+1}(f) & =\frac{1}{8} \int \frac{1}{f} \sum_{i=1}^{N}\left(\frac{\partial f}{\partial x_{i}}-\frac{\partial f}{\partial x_{i+1}}\right)^{2} \Phi_{N} d x \\
& =\frac{1}{8} \sum_{i=1}^{N}\left(\lambda_{i}-\lambda_{i+1}\right)^{2}=\frac{1}{N} \int\left(\frac{\partial \bar{\lambda}}{\partial \theta}\right)^{2} d \theta \leqq \frac{C}{8 N}
\end{aligned}
$$

On the other hand if $f=c \exp \Sigma \lambda_{i} x_{i}$, one can then calculate explicitly and show that for any function $F\left(x_{-k}, \ldots, x_{k}\right)$ depending on $(2 k+1)$ coordinates $(1 / N) \Sigma J(i / N) F\left(x_{-k+i}, \ldots, x_{k+i}\right)$ is also almost a constant relative to our Gibbs state and compute the constant explicitly in terms of $\bar{m}(\theta)$. The aim of this section is to show that the above consequence for local averages follows from entropy bounds of the type (4.1). This will be the key step of our main theorem.

We will be dealing with sets of probability measures or densities $A_{N, C_{1}, C_{2}}$ defined in terms of two constants $C_{1}, C_{2}$ but in dimensions $N$, that vary. The purpose will be to obtain for these classes certain results that are valid uniformly in $N$ so long as $C_{1}$ and $C_{2}$ are held fixed.

Let $\underline{A}_{N, C_{1}, C_{2}}$ be the class of densities $f$ on $R^{N}$ satisfying the following bounds:

$$
\begin{aligned}
\frac{1}{N} \int\left[\Sigma \omega\left(x_{i}\right)\right] f \Phi_{N} d x & \leqq C_{1}, \\
\sum_{j=1}^{N} \hat{I}_{j, j+1}(f) & \leqq \frac{C_{2}}{N} .
\end{aligned}
$$

Here $\omega(\cdot)$ is the function defined in (1.4).

Let $F$ be a function depending on the $2 k+1$ variables $\left\{x_{j}:|j| \leqq k\right\}$ on $R^{2 k+1}$ which is bounded and continuous on $R^{2 k+1}$. Given such a function let us define $\widetilde{F}(y)$ for $y \in R$ by

$$
\tilde{F}(y)=\frac{1}{[M(\lambda)]^{2 k+1}} \int \exp \left[\lambda \Sigma x_{j}\right] F\left(x_{-k}, \ldots, x_{k}\right) \Phi_{2 k+1} d x,
$$

with $\lambda=h^{\prime}(y)$. Let us define for any integer $i \in[1,2, \ldots, N]$ and $l$ the quantities $\xi_{i}=F\left(x_{i-k}, \ldots, x_{i+k}\right)$ and $\tilde{\xi}_{i, l}=\tilde{F}\left((1 / 2 l+1)\left(x_{i-l}+\cdots+x_{i+l}\right)\right)$. Finally let $J(\cdot)$ be a smooth function of $\theta \in S$. The main result of this section is the following theorem.

Theorem 4.1. For any given constant $C_{1}$ and $C_{2}$, integer $k$ and choices of $F(\cdot)$ and $J(\cdot)$,

$$
\lim _{\varepsilon \rightarrow 0} \lim \sup _{N \rightarrow \infty} \sup _{f \in A_{N, C_{1}, C_{2}}} \int \frac{1}{N} \mid \Sigma\left[J\left(\frac{i}{N}\right)\left(\xi_{i}-\bar{\xi}_{i,[N \varepsilon]}\right) \mid f \Phi_{N} d x=0 .\right.
$$

We will first prove some auxiliary lemmas and theorems before finally returning to the proof of Theorem 4.1. The first step is Theorem 4.2 which we now state and prove.

Theorem 4.2. For any $C_{1}, C_{2}$ and $F(\cdot)$,

$$
\lim _{l \rightarrow \infty} \limsup \sup _{N \rightarrow \infty} \int \frac{1}{N} \sum_{j=1}^{N}\left|\frac{1}{2 l+1} \sum_{i=j, C_{1}, C_{2}}^{j+l} \xi_{i}-\tilde{\xi}_{j, l}\right| f \Phi_{N} d x=0 .
$$


Proof. At first glance the above theorem looks hard because $N$ is becoming large and things can get out of control. We will show however that the hard work has all been done already and we can obtain (4.6) from our earlier results in Sects. 2 and 3 .

First Step. Since $F$ is a bounded function and $l$ is becoming large while $k$ remains fixed we can replace $(1 / 2 l+1) \sum_{i=j-l}^{j+l} \xi_{i}$ by $(1 / 2 l-2 k+1) \sum_{i=j-l+k}^{j+l-k} \xi_{i}$, and the error will be uniformly small.

Second Step. If we denote by $\psi_{l}\left(y_{-l}, \ldots, y_{l}\right)$ the function

$$
\psi_{l}\left(y_{-l}, \ldots, y_{l}\right)=\frac{1}{2(l-k)+1} \sum_{i=-(l-k)}^{(l-k)} F\left(y_{i-k}, \ldots, y_{i+k}\right)-\tilde{F}\left(\frac{y_{-l}+\cdots+y_{l}}{2 l+1}\right),
$$

then

$$
\frac{1}{N} \int \sum_{j=1}^{N}\left|\frac{1}{2 l+1} \sum_{i=j-l}^{j+l} \xi_{i}-\bar{\xi}_{j, l}\right| f \Phi_{N} d x=\int\left|\psi_{l}\left(y_{-l}, \ldots, y_{l}\right)\right| f^{l} \Phi_{2 l+1} d y
$$

[see Lemma 2.2 for the definition of $f^{l}$ ]. If we denote by $\underline{B}_{N, C_{1}, C_{2}}^{l}$ the range of $f^{l}$ as $f$ varies over $\underline{A}_{N, C_{1}, C_{2}}$, then

$$
\sup _{f \in \underline{A}_{N, C_{1}, C_{2}}} \int \frac{1}{N} \sum_{j=1}^{N}\left|\frac{1}{2 l+1} \sum_{i=j+l}^{j-l} \xi_{i}-\tilde{\xi}_{i, l}\right| f \Phi_{N} d x=\sup _{y \in \underline{B}_{N, C_{1}, C_{2}}^{l}} \int\left|\psi_{l}\right| g \Phi_{2 l+1} d y .
$$

Now $\underline{B}_{N, C_{1}, C_{2}}^{l}$ are all distributions on $R^{2 l+1}$. We will verify that $\underline{B}_{N, C_{1}, C_{2}}^{l}$ is a tight family on $R^{2 l+1}$ as $N \rightarrow \infty$ and obtain information about its limit points $\underline{B}_{C_{1}, C_{2}}^{l}$.

From (4.2) we obtain

$$
\frac{1}{2 l+1} \int\left[\sum_{i=-l}^{l} \omega\left(y_{i}\right)\right] g \Phi_{2 l+1}(y) d y \leqq C_{1} \text { for all } g \in \underline{B}_{N, C_{1}, C_{2}}^{l} .
$$

This implies tightness immediately. If $\beta$ is any element of $\underline{B}_{C_{1}, C_{2}}^{l}$, by lower semicontinuity and (4.7) we obtain

$$
\sup _{\beta \in \underline{B}_{C_{1}, C_{2}}^{l}} \frac{1}{2 l+1} \int \Sigma \omega\left(y_{i}\right) d \beta \leqq C_{1} .
$$

Further the function

$$
I_{l}(\beta)=\sum_{j=-l}^{l-1} \hat{I}_{j, j+1}(\beta)
$$

is lower semicontinuous. From (4.3), Lemma 2.2 and lower semicontinuity we find that $I_{l}(\beta)=0$ for $\beta \in \underline{B}_{C_{1}, C_{2}}^{l}$.

Third Step. We can now replace the limsup sup in (4.6) by sup. Moreover if $\beta \in \underline{B}_{C_{1}, C_{2}}^{l}$,

$$
N \rightarrow \infty \quad f \in \underline{A}_{N, C_{1}, C_{2}} \quad \beta \in \underline{\underline{C}}_{C_{1}, C_{2}}^{l}
$$

$$
\frac{1}{2 l+1} \int\left[\Sigma \omega\left(y_{i}\right)\right] d \beta \geqq \frac{1}{2 l+1} \int\left[\Sigma\left|y_{i}\right|\right] d \beta
$$


It is therefore sufficient to prove that

where

$$
\lim _{l \rightarrow \infty} \sup _{\beta \in \Gamma_{l, C_{1}}} \int\left|\psi_{l}\right| d \beta=0
$$

$$
\Gamma_{l, C_{1}}=\left\{\beta: \frac{1}{2 l+1} \int\left[\Sigma\left|y_{i}\right|\right] d \beta \leqq C_{1}, I_{l}(\beta)=0\right\} .
$$

Final Step. If we define the generator

$$
L^{[l]}=\frac{1}{2} \sum_{i=-l}^{l-1}\left(\frac{\partial}{\partial y_{i}}-\frac{\partial}{\partial y_{i+1}}\right)^{2}-\frac{1}{2} \sum_{i=-l}^{l-1}\left(\phi^{\prime}\left(y_{i}\right)-\phi^{\prime}\left(y_{i+1}\right)\right)\left(\frac{\partial}{\partial y_{i}}-\frac{\partial}{\partial y_{i+1}}\right),
$$

then $I_{l}(\beta)=0$ implies that $\beta$ is an invariant measure for the diffusion on $R^{2 l+1}$ with generator $L^{[l]}$. We know that these are convex combinations of the ergodic ones concentrated on the various hyperplanes $(1 / 2 l+1) \sum_{|j| \leqq l} y_{j}=y$. Therefore

$$
\beta=\int v_{y}^{(2 l+1)} d \hat{\beta}
$$

where $\hat{\beta}$ is the distribution of $y$ under $\beta$. From (4.9) it follows that $\int|y| d \hat{\beta} \leqq$ $(1 / 2 l+1) \int\left(\Sigma\left|y_{i}\right|\right) d \beta \leqq C_{1}$. One can now obtain (4.8) from Theorem 3.5 and the above bound.

If we take a look at what has been achieved in Theorem 4.2 for a "Local Gibbs state," i.e. a state satisfying (4.2) and (4.3) for some constants $C_{1}$ and $C_{2}$, then relative to such a probability distribution one can always replace $(1 / N) \Sigma J(i / N) \xi_{i}$ by $(1 / N) \Sigma J(i / N)\left((1 / 2 l+1) \sum_{j=i-l}^{i+l} \xi_{i}\right)$, and according to Theorem 4.2 this can be replaced asymptotically by $(1 / N) \Sigma J(i / N) \widetilde{\xi}_{i, l}$, provided $l$ is large and fixed. However Theorem 4.1 demands that $l$ be chosen as $[N \varepsilon]$ for arbitrarily small but fixed $\varepsilon>0$. The next two theorems are to make the replacement possible.

Theorem 4.3. For any $\delta>0$ and $C_{1}$ and $C_{2}$ finite

$$
\begin{aligned}
& \lim _{\varepsilon \rightarrow 0} \limsup _{l \rightarrow \infty} \limsup _{N \rightarrow \infty} \sup _{2 l+1 \leqq|m| \leqq \in N} \sup _{f \in A_{N, C_{1}, C_{2}}} \\
& \frac{1}{N} \int\left[\# i:\left|\frac{x_{i-l}+x_{i+l}}{2 l+1}-\frac{x_{i+m-l}+x_{i+m+l}}{2 l+1}\right| \geqq \delta\right] f \Phi_{N} d x=0 .
\end{aligned}
$$

Proof. The proof is very much like the proof of Theorem 4.2. We first rewrite the integral through the projections $\alpha^{m . l}$ introduced before Lemma 2.3. Let us note that the measure $\alpha$ and the density $f$ will be used interchangeably. The integral then is

$$
\alpha^{m, l}\left[\left(y_{j}, z_{j}\right):\left|\frac{1}{2 l+1} \Sigma y_{j}-\frac{1}{2 l+1} \Sigma z_{j}\right| \geqq \delta\right] .
$$

First Step. Let us denote by $\underline{B}_{N, C_{1}, C_{2}}^{m, l}$ the range of $f^{m, l}$ as $\alpha$ or $f$ varies over $\underline{A}_{N, C_{1}, C_{2}}$. These are probability measures on $R^{2 l+1} \times R^{2 l+1}$ with coordinates $\left\{y_{j}:|j| \leqq l\right\}$ and $\left\{z_{j}:|j| \leqq l\right\}$. We denote by $\underline{B}_{C_{1}, C_{2}}^{l, \varepsilon}$ the set of limit points of $\sum_{2 l+1 \leqq|m| \leqq N \varepsilon} \underline{B}_{N, C_{1}, C_{2}}^{m, l}$. The basic estimate (4.2) yields for any $g \in \underline{B}_{N, C_{1}, C_{2}}^{m, l}$, 


$$
\frac{1}{4 l+2} \int\left[\Sigma \omega\left(y_{i}\right)+\Sigma \omega\left(z_{i}\right)\right] g \Phi_{4 l+2} d y d z \leqq C_{1},
$$

and thereby tightness. The lower bound on the gap $|m| \geqq 2 l+1$ is to make sure that the two sets of blocks of size $2 l+1$ with their centers separated by $m$ have no common sites. If $\beta$ is any limit point in $\underline{B}_{C_{1}, C_{2}}^{l, \varepsilon}$ we obtain the following estimates:

$$
\begin{gathered}
\frac{1}{4 l+2} \int\left[\Sigma \omega\left(y_{i}\right)+\Sigma \omega\left(z_{i}\right)\right] d \beta \leqq C_{1}, \\
\sum_{i=-l}^{l-1} \hat{I}_{i, i+1}^{Y}(\beta)=0, \\
\sum_{i=-l}^{l} \hat{I}_{i, i+1}^{Z}(\beta)=0, \\
\hat{I}^{Y . Z}(\beta) \leqq C_{2} \varepsilon^{2} .
\end{gathered}
$$

These are obtained from lower semicontinuity and Lemma 2.3.

Second Step. (4.14) and (4.15) imply that the measure $\beta$ on $R^{2 l+1} \times R^{2 l+1}$ is invariant with respect to the diffusions

$$
L^{Y}=\frac{1}{2} \sum_{i=-l}^{l-1}\left(\frac{\partial}{\partial y_{i}}-\frac{\partial}{\partial y_{i+1}}\right)^{2}-\frac{1}{2} \sum_{i=-l}^{l-1}\left(\phi^{\prime}\left(y_{i}\right)-\phi^{\prime}\left(y_{i+1}\right)\right)\left(\frac{\partial}{\partial y_{i}}-\frac{\partial}{\partial y_{i+1}}\right),
$$

and a similar one involving the second set of coordinates. This implies that

$$
\beta(d y, d z)=\int v_{a}^{(2 l+1)}(d y) v_{b}^{(2 l+1)}(d z) \hat{\beta}(d a, d b),
$$

where $\hat{\beta}$ is the distribution of $a=(1 / 2 l+1) \Sigma y_{i}$ and $b=(1 / 2 l+1) \Sigma z_{i}$ on $R^{2}$. Here $v$ is as defined in Theorem 3.5.

Third Step. From (4.3) for $\beta$ one can clearly obtain

$$
\int[|a|+|b|] \hat{\beta}(d a, d b) \leqq 2 C_{1} .
$$

According to Lemma 4.4 the inequality (4.16) provides an estimate

where

$$
\bar{I}^{(l)}(\widehat{\beta}) \leqq C_{2} \varepsilon^{2}(2 l+1)^{2},
$$

$$
\bar{I}^{(l)}(\widehat{\beta})=\sup _{u>0} \int\left[-\frac{G^{(l)} u}{u}\right] d \hat{\beta}(a, b),
$$

and $G^{(l)}$ is the generator of the diffusion

$$
G^{(l)} u=\frac{1}{2}\left(\frac{\partial}{\partial a}-\frac{\partial}{\partial b}\right)^{2} u-\frac{1}{2}\left(h_{2 l+1}^{\prime}(a)-h_{2 l+1}^{\prime}(b)\right)\left(\frac{\partial u}{\partial a}-\frac{\partial u}{\partial b}\right)
$$

on $R^{2}$. Here $h_{2 l+1}(a)=-\log \psi_{2 l+1}(a)$ and $\psi_{k}(a)$ is the density of $x_{1}+\cdots+x_{k} / k$, where $x_{1}, \ldots, x_{k}$ are independent with common density $e^{-\phi(x)}$.

Let us consider the range $\Gamma_{C_{1}, C_{2}}^{l, \varepsilon}$ of $\hat{\beta}$ on $R^{2}$ as $\beta$ varies over $\underline{B}_{C_{1}, C_{2}}^{l, \varepsilon}$. Then $\hat{\beta}$ satisfies (4.18) and (4.19). We now consider the set of limit points of $\Gamma_{C}^{l, \varepsilon}$ as $l \rightarrow \infty$ and denote it by $\Gamma_{C_{1}, C_{2}}^{\varepsilon}$. 
We have tightness from (4.18) and Lemma 4.5 gives us the estimate

$$
\int\left[h^{\prime}(a)-h^{\prime}(b)\right]^{2} d \widehat{\beta}(a, b) \leqq 8 C_{2} \varepsilon^{2}
$$

for every $\hat{\beta} \in \Gamma_{C_{1}, C_{2}}^{\varepsilon}$. We also have the companion estimate: for every $\hat{\beta} \in \Gamma_{C_{1}, C_{2}}^{\varepsilon}$,

$$
\frac{1}{2} \int[|a|+|b|] d \hat{\beta}(a, b) \leqq C_{1} .
$$

Finally if we let $\varepsilon \rightarrow 0$ and again look at the limit points, denoting the set by $\Gamma_{C_{1}, C_{2}}^{0}$, then

$$
\int\left[h^{\prime}(a)-h^{\prime}(b)\right]^{2} d \widehat{\beta}(a, b)=0
$$

for every $\hat{\beta} \in \Gamma_{C_{1}, C_{2}}^{0}$. From the strict convexity of $h, h^{\prime}$ is a strictly monotone map and $\beta$ is then concentrated on the diagonal of $R^{2}$. But this is precisely the content of the theorem because of (4.11).

We now have two lemmas to prove in order to complete the proof of Theorem 4.3:

Lemma 4.4. Let $\beta$ be a probability measure on $R^{2 l+1} \times R^{2 l+1}$ of the form

$$
\beta(d y, d z)=\int v_{a}^{(2 l+1)}(d y) v_{b}^{(2 l+1)}(d z) \hat{\beta}(d a, d b),
$$

where $v_{a}^{(2 l+1)}$ is the restriction of $\Phi_{2 l+1}$ to the hyperplane of average charge a. Then

$$
\bar{I}^{(2 l+1)}(\hat{\beta}) \leqq(2 l+1)^{2} I^{Y, Z}(\beta),
$$

where $\bar{I}$ is defined in (4.20) and $I^{Y . Z}$ in Lemma 2.3.

Proof. We recall that $e^{-h_{2 l+1}(a)}$ is the density at $a$ of the mean $\left(x_{1}+\cdots+x_{2 l+1}\right) /$ $(2 l+1)$, where $x_{1}, \ldots, x_{2 l+1}$ are independent, all having $e^{-\phi(x)}$ as density. We notice first that

$$
\begin{aligned}
& \int g(a) e^{-h_{2 l+1}(a)} \int \phi^{\prime}\left(y_{0}\right) v_{a}^{(2 l+1)}(d y) d a \\
& \quad=\int g\left(\frac{1}{2 l+1} \Sigma y_{j}\right) \phi^{\prime}\left(y_{0}\right) e^{-\Sigma \phi\left(y_{j}\right)} d y d a \\
& \quad=\frac{1}{2 l+1} \int g^{\prime}\left(\frac{1}{2 l+1} \Sigma y_{j}\right) e^{-\Sigma \phi\left(y_{j}\right)} d y d a=\frac{1}{2 l+1} \int g^{\prime}(a) e^{-h_{2 l+1}(a)} d a \\
& =\frac{1}{2 l+1} \int g(a) h_{2 l+1}^{\prime}(a) e^{-h_{2 l+1}(a)} d a .
\end{aligned}
$$

Since $g$ is arbitrary and $v_{a}$ and $h_{2 l+1}^{\prime}(a)$ are continuous in $a$, we obtain

where

$$
\begin{aligned}
& \int \phi^{\prime}\left(y_{0}\right) v_{a}^{(2 l+1)}(d y)=\frac{1}{2 l+1} h_{2 l+1}^{\prime}(a), \\
& I^{Y, Z}(\beta)=\sup _{u>0}\left[-\int\left(\frac{G u}{u}\right) \beta(d u, d z)\right],
\end{aligned}
$$

$$
G=\frac{1}{2}\left(\frac{\partial}{\partial y_{0}}-\frac{\partial}{\partial z_{0}}\right)^{2}-\frac{1}{2}\left[\phi^{\prime}\left(y_{0}\right)-\phi^{\prime}\left(z_{0}\right)\right]\left(\frac{\partial}{\partial y_{0}}-\frac{\partial}{\partial z_{0}}\right) .
$$


If $\beta=\int v_{a}^{(2 l+1)}(d y) v_{b}^{(2 l+1)}(d z) \hat{\beta}(d a, d b)$, we can take $u$ to be a function of $a$ and $b$ of the form $u\left((1 / 2 l+1) \Sigma y_{j},(1 / 2 l+1) \Sigma z_{j}\right)$. Then

$$
\begin{aligned}
I^{Y, Z}(\beta) \geqq & \sup _{u}\left[-\int \frac{1}{u}\left[\frac{1}{2} \cdot\left(\frac{1}{2 l+1}\right)^{2}\left(\frac{\partial}{\partial a}-\frac{\partial}{\partial b}\right)^{2} u\right.\right. \\
& \left.\left.-\frac{1}{2(2 l+1)}\left(\phi^{\prime}\left(y_{0}\right)-\phi^{\prime}\left(z_{0}\right)\right)\left(u_{a}-u_{b}\right)\right] \beta(d y d z)\right] .
\end{aligned}
$$

If we now use (4.25),

$$
\begin{aligned}
I^{Y, Z}(\beta) \geqq & \frac{1}{(2 l+1)^{2}}\left[\operatorname { s u p } _ { u } \left\{-\int \frac{1}{2 u}\left[\left(\frac{\partial}{\partial a}-\frac{\partial}{\partial b}\right)^{2} u\right.\right.\right. \\
& \left.-\left(h_{2 l+1}(a)-h_{2 l+1}(b)\right)\left(u_{a}-u_{b}\right)\right] \hat{\beta} d a d b \\
= & \frac{1}{(2 l+1)^{2}} \bar{I}^{\prime}(\hat{\beta}) .
\end{aligned}
$$

Lemma 4.5. If $\left\{\hat{\beta}_{l}\right\}$ is a sequence of probability measures on $R^{2}$ satisfying

and

$$
\int[|a|+|b|] \widehat{\beta}_{l}(d a, d b) \leqq 2 C_{1}
$$

and if $\hat{\beta}$ is any limit point of $\hat{\beta}_{l}$, then

$$
I^{(l)}\left(\hat{\beta}_{l}\right) \leqq C_{2}(2 l+1)^{2} \varepsilon^{2},
$$

$$
\int\left[h^{\prime}(a)-h^{\prime}(b)\right]^{2} \hat{\beta}(d a, d b) \leqq 8 C_{2} \varepsilon^{2} .
$$

Proof. (4.27) implies for every $u$

$$
\begin{aligned}
& \frac{1}{2}\left[\int \frac{1}{u}\left[\left(\frac{\partial}{\partial a}-\frac{\partial}{\partial b}\right)^{2} u-\left(h_{2 l+1}^{\prime}(a)-h_{2 l+1}^{\prime}(b)\right)\left(u_{a}-u_{b}\right)\right] \hat{\beta}_{l}(d a, d b)\right] \\
& \quad \geqq-C \varepsilon^{2}(2 l+1)^{2} .
\end{aligned}
$$

Taking $u=\exp [(2 l+1) v(a, b)]$ for some $v$,

$$
\begin{aligned}
& {\left[\int \left\{(2 l+1)\left(\frac{\partial}{\partial a}-\frac{\partial}{\partial b}\right)^{2} v+(2 l+1)^{2}\left(\frac{\partial}{\partial a}-\frac{\partial}{\partial b}\right)^{2}\right.\right.} \\
& \left.\left.\quad-(2 l+1)\left(h_{2 l+1}^{\prime}(a)-h_{2 l+1}^{\prime}(b)\right)\left(\frac{\partial v}{\partial a}-\frac{\partial v}{\partial b}\right) \hat{\beta}_{l}(d a, d b)\right\}\right] \geqq-2 C \varepsilon^{2}(2 l+1)^{2} .
\end{aligned}
$$

If $v$ is a constant outside a compact set one can divide (4.27) by $(2 l+1)$ and let $l \rightarrow \infty$ to obtain

$$
\int\left[\left(\frac{\partial v}{\partial a}-\frac{\partial v}{\partial b}\right)^{2}-\left(h^{\prime}(a)-h^{\prime}(b)\right)\left(\frac{\partial v}{\partial a}-\frac{\partial v}{\partial b}\right)\right] \beta(d a, d b) \geqq-2 C \varepsilon^{2} .
$$

We are done if we let

$$
v(a, b)=\frac{1}{2}[(h(a)+h(b)]
$$


except, then $v$ is not constant outside a compact set. But we can take

$$
v_{\tau}(a, b)=\min \left[\frac{1}{2}[h(a)+h(b)], \tau\right],
$$

and let $\tau \rightarrow \infty . v_{\tau}$ is not quite smooth enough. But one can mollify $v_{\tau}$ somewhat.

We now prove a slightly strengthened version of Theorem 4.3.

Theorem 4.6. For any given finite constants $C_{1}, C_{2}$,

$$
\begin{aligned}
& \lim _{\varepsilon \rightarrow 0} \limsup _{l \rightarrow \infty} \limsup _{N \rightarrow \infty} \sup _{2 l+1 \leqq|m| \leqq \Sigma N} \sup _{f \in A_{N, C_{1}, C_{2}}} \\
& \quad \int\left\{\frac{1}{N} \Sigma\left|\frac{x_{i+l}+\cdots+x_{i+l}}{2 l+1}-\frac{x_{i+m-k}+\cdots+x_{i+m+k}}{2 l+1}\right|\right\} f \Phi_{N} d x=0 .
\end{aligned}
$$

Proof. Given Theorem 4.5 all we need to derive Theorem 4.6 is some uniform integrability of $(1 / 2 l+1) \sum_{|l| \leq l}\left|y_{j}\right|$ relative to all the distributions $\alpha^{l}$ with $\alpha$ varying over $A_{N, C_{1}, C_{2}}$. But this is precisely the role of condition (4.2) especially with $\omega(x)$ satisfying (1.4).

Finally we prove

Theorem 4.7. For any $C_{1}, C_{2}$,

$$
\begin{aligned}
& \lim _{\varepsilon \rightarrow 0} \limsup _{l \rightarrow \infty} \limsup _{N \rightarrow \infty} \sup _{t \in A_{N, C_{1}, C_{2}}} \\
& \quad \int \frac{1}{N} \Sigma\left|\frac{x_{i-l}+\cdots+x_{i+l}}{2 l+1}-\frac{x_{i-[N \varepsilon]}+\cdots+x_{i+[N \varepsilon]}}{2[N \varepsilon]+1}\right| f \Phi_{N} d x=0 .
\end{aligned}
$$

Proof. Theorem 4.7 follows immediately from Theorem 4.6. An average over a long block of length $2[N \varepsilon]+1$ is just an average of averages over short blocks of length $(2 l+1)$. According to Theorem 4.6 they do not differ by much among themselves so the average is close to any one of them.

Theorem 4.7 allows us to deduce Theorem 4.1 from Theorem 4.2. One uses the continuity of the function $\tilde{F}(y)$. Any trouble arising from large values of $\left(x_{i-l}+\cdots+x_{i+l} / l\right)[\tilde{F}(y)$ need not be uniformly continuous $]$ is taken care of by (4.2) which tells us that there cannot be too many such blocks.

\section{Hydrodynamic Limit}

Let us start our evolution with an initial distribution $f_{N}^{0}$ satisfying (1.9) and (1.11). We will study the evolution in a fixed interval $[0, T]$. In fact without loss of generality we will assume that $T=1$. With $f_{N}^{0}$ as initial distribution we have a measure $P_{N}$ on the space $C\left\{[0,1]: R^{N}\right\}$ of trajectories $\left\{x_{j}(t): 0 \leqq t \leqq 1,1 \leqq j \leqq N\right\}$. Let us denote by $M_{l}$ the compact metric space (under weak convergence) of all signed measures on $S$ of total variation at most $l$. The space $M=U_{l} \underline{M}_{l}$ is viewed mainly as a measurable space. We denote by $\Omega_{l}$ the space $C\left\{[0,1] ; M_{l}\right\}$ of all weakly continuous maps of $[0,1]$ into $M_{l} . \Omega_{l}$ is a complete separable metric space with uniform convergence in weak topology. The space $\Omega=U_{l} \Omega_{l}$ is again viewed only as a measurable space. Given a collection of trajectories $\left\{x_{j}(t)\right\}$ we can 
associate a point in $\Omega$ by

$$
\mu_{N}(t)=\frac{1}{N} \Sigma x_{j}(t) \dot{\delta}_{j / N}
$$

This introduces a measure $Q_{N}$ on $\Omega$ as the distribution of $\mu_{N}(\cdot)$, where $\left\{x_{j}(\cdot)\right\}$ have $P_{N}$ for their distribution. According to Lemmas 6.1 and 6.2, from any subsequence of $Q_{N}$ we can choose a further subsequence that converges weakly on $\Omega$. What this means is that for any bounded function $F$ whose restriction to $\Omega_{l}$ is continuous for every $l$, we have convergence of expectations. Let $Q$ be any limit point along any subsequence. The main theorem of the section is the following:

Theorem 5.1. Let $Q$ be any limit point. Then

a) $Q[\mu(\cdot): \mu(t)=m(t, \theta) d \theta$ a.e. $t]=1$,

b) $Q\left[\mu(\cdot): \mu(0)=m_{0}(\theta) d \theta\right]=1$,

c) $Q\left[\mu(\cdot): \int_{0}^{1} \int_{S} h(m(t, \theta)) d \theta d t<\infty\right]=1$,

d) $Q\left[\mu(\cdot): \int_{0}^{1} \int_{S}\left\{\left[h^{\prime}(m(t, \theta))\right]_{\theta}\right\}^{2} d \theta d t<\infty\right]=1$,

e) $Q\left[\mu(\cdot): \int_{S} J(\theta) \mu(t, d \theta)-\int_{S} J(\theta) \mu(0, d \theta)=\frac{1}{2} \int_{0}^{t} \int_{S} J^{\prime \prime}(\theta) h^{\prime}(m(s, \theta)) d \theta d s\right]=1$.

Proof. From the monotonicity of the entropy (see Eq. 2.4) and Lemma 6.3 we conclude that for each $t>0, Q[\mu: \mu(t, d \theta)=m(t, \theta) d \theta]=1$ and $\int_{S} h(m(t, \theta)) d \theta \leqq C$ for all $t \geqq 0$. Now Fubini's theorem will yield a) as well as c). b) is just a restatement of condition (1.9). To prove d) we use Fubini's theorem and Lemma 6.6. We note that

$$
E^{Q}\left[\int_{0}^{1} \int_{S}\left\{\left[h^{\prime}(m(\theta, t))\right]_{\theta}\right\}^{2} d \theta d t\right]=E^{\tilde{Q}}\left[\int_{S}\left\{\left[h^{\prime}(m(\theta))\right]_{\theta}\right\}^{2} d \theta\right]
$$

where $\tilde{Q}=\int_{D}^{1} Q_{t} d t$ and $Q_{t}$ is the marginal at time $t$ of $Q$. Lemma 6.6 of course applies to $\widetilde{Q}$.

We now turn to the proof of e). Let us consider the cutoff function $\psi_{l}$ of Lemma 6.4 and pick $\varepsilon>0$. We then consider for some $0<t \leqq 1$,

$$
\begin{aligned}
F_{l, \varepsilon}^{N, t}(\mu(\cdot))= & \int_{S} J(\theta) \mu(t, d \theta)-\int_{s} J(\theta) \mu(0, d \theta) \\
& -\frac{1}{2 N} \int_{0}^{t} \sum_{j=1}^{N} J^{\prime \prime}\left(\frac{j}{N}\right) \tilde{\psi}_{l}\left(\frac{x_{j-[N \varepsilon]}(s)+\cdots+x_{j+[N \varepsilon]}(s)}{1+[2 N \varepsilon]}\right) d s .
\end{aligned}
$$

As functionals $F_{l, \varepsilon}^{N, t}(\mu(\cdot))$ converge as $N \rightarrow \infty$ to a limit given by

$$
\begin{aligned}
F_{l, \varepsilon}^{t}(\mu(\cdot))= & \int_{s} J(\theta) \mu(t, d \theta)-\int_{s} J(\theta) \mu(O m, d \theta) \\
& -\int_{0}^{t} \int_{s} J^{\prime}(\theta) \tilde{\psi}_{l}\left(\frac{\mu(s,[\theta-\varepsilon, \theta+\varepsilon])}{2 \varepsilon}\right) d \theta d s,
\end{aligned}
$$


and the convergence is uniform on compact subsets. Therefore

$$
E^{Q}\left[\left|F_{l, \varepsilon}^{t}(\mu(\cdot))\right|\right] \leqq \varlimsup_{N \rightarrow \infty} E^{Q_{N}}\left[\left|F_{l, \varepsilon}^{N, t}(\mu(\cdot))\right|\right] .
$$

Let us look at the right-hand side first. By Itô's formula

$$
\begin{aligned}
\frac{1}{N} & \Sigma J\left(\frac{i}{N}\right) x_{i}(t)-\frac{1}{N} \Sigma J\left(\frac{i}{N}\right) x_{i}(0) \\
& =\frac{N^{2}}{2} \int_{0}^{t} \frac{1}{N} \Sigma J\left(\frac{i}{N}\right)\left\{\phi^{\prime}\left(x_{i-1}(s)\right)-2 \phi^{\prime}\left(x_{i}(s)\right)+\phi^{\prime}\left(x_{i+1}(s)\right)\right\} d s+M_{N}(t) \\
& =\frac{N^{2}}{2 N} \int_{0}^{t} \Sigma\left(J\left(\frac{i+1}{N}\right)-2 J\left(\frac{i}{N}\right)+J\left(\frac{i-1}{N}\right)\right) \phi^{\prime}\left(x_{i}(s)\right) d s+M_{N}(t),
\end{aligned}
$$

where $M_{N}(t)$ is a Martingale and an explicit calculation yields

$$
E^{P_{N}}\left[\left|M_{N}(t)\right|^{2}\right]=\frac{1}{N^{2}} N^{2} t \Sigma\left[J\left(\frac{i+1}{N}\right)-J\left(\frac{i}{N}\right)\right]^{2}=\frac{C t}{N},
$$

and tends to zero with $N$. Moreover because of Lemmas 6.4 and (5.3),

$$
E^{P_{N}}\left[\left\lfloor\frac{1}{N} \Sigma J\left(\frac{i}{N}\right) x_{i}(t)-\frac{1}{N} \Sigma J\left(\frac{i}{N}\right) x_{i}(0)-\frac{1}{2 N} \int_{0}^{t} \Sigma J^{\prime \prime}\left(\frac{i}{N}\right) \phi^{\prime}\left(x_{i}(s)\right) d s \mid\right] \rightarrow 0 .\right.
$$

Let us consider

$$
E^{P_{N}}\left|\frac{1}{2 N} \int_{0}^{t} \Sigma J^{\prime \prime}\left(\frac{i}{N}\right) \phi^{\prime}\left(x_{i}(s)\right) d s-\frac{1}{2 N} \int_{0}^{t} \Sigma J^{\prime \prime}\left(\frac{i}{N}\right) \psi_{l}\left(x_{i}(s)\right) d s\right|=\Delta_{N}(l) .
$$

According to Lemma 6.4,

$$
\lim _{l \rightarrow \infty} \limsup _{N \rightarrow \infty} \Delta_{N}(l)=0
$$

Let us consider

$$
\begin{aligned}
E^{P_{N}} & \mid \frac{1}{2 N} \int_{0}^{t} \Sigma J^{\prime \prime}\left(\frac{i}{N}\right) \psi_{l}\left(x_{i}(s) d s\right. \\
& -\frac{1}{2 N} \int_{0}^{t} \Sigma J^{\prime \prime}\left(\frac{i}{N}\right) \tilde{\psi}_{l}\left(\frac{x_{i-N \varepsilon}(s)+\cdots+x_{i+N \varepsilon}(s)}{2 N \varepsilon+1}\right) d s \mid=\bar{\Delta}_{N}(l, \varepsilon) .
\end{aligned}
$$

According to Theorem 4.1 and then (2.4),

$$
\lim _{\varepsilon \rightarrow 0} \varlimsup_{N \rightarrow \infty} \bar{\Delta}_{N}(l, \varepsilon)=0 \text { for every } l \text {. }
$$

If we combine (5.4), (5.5) and (5.6) we obtain

$$
\lim _{l \rightarrow \infty} \limsup _{\varepsilon \rightarrow 0} \limsup _{N \rightarrow \infty} E^{Q_{N}}\left[\left|F_{l, \varepsilon}^{N, t}(\mu(\cdot))\right|\right]=0,
$$


and combined with (5.2) this yields

$$
\lim _{l \rightarrow \infty} \lim _{\varepsilon \rightarrow 0} E^{Q}\left[\left|F_{l, \varepsilon}^{t}(\mu(\cdot))\right|\right]=0 .
$$

We can let $\varepsilon \rightarrow 0$ inside because $F_{l, \varepsilon}^{t}$ is uniformly bounded and obtain using a)

where

$$
\lim _{l \rightarrow \infty} E^{Q}\left[\left|F_{l}^{t}(\mu(\cdot))\right|\right]=0
$$

$$
F_{l}^{t}(\mu(\cdot))=\langle J, \mu(t)\rangle-\langle J, \mu(0)\rangle-\frac{1}{2} \int_{0}^{t} \int_{s} J^{\prime \prime}(\theta) \tilde{\psi}_{l}(m(s, \theta)) d s d \theta
$$

Now we let $l \rightarrow \infty$. We can use Lemmas 6.5 and 6.3 and the dominated convergence theorem to conclude that

$$
E^{Q}\left[\left|F^{t}(\mu(\cdot))\right|\right]=0
$$

where

$$
F^{t}(\mu(\cdot))=\langle J, \mu(t)\rangle-\langle J, \mu(0)\rangle-\frac{1}{2} \int_{0}^{t} \int_{s} J \cdot(\theta) h^{\prime}(m(s, \theta)) d s d \theta .
$$

This proves the theorem.

Now to complete the proof of Theorem 1.1 all we need is to appeal to a uniqueness theorem for weak solutions of

$$
\frac{\partial m}{\partial t}=\frac{1}{2}\left[h^{\prime}(m(t, \theta))\right]_{\theta \theta},\left.\quad m(t, \theta)\right|_{t=0}=m_{0}(\theta)
$$

\section{Auxiliary Lemmas}

In this section we shall prove some lemmas that were used in Sect. 5 where the hydrodynamic limit was identified. The first two lemmas show compactness of the process $\mu_{N}(t)$ defined by $(5.1)$, in the sense described in that section. Lemma 6.3 is essentially (a) of Theorem 5.1 stated in a general way. Lemmas 6.4 and 6.5 are used in implementing the truncation in (c) of Theorem 5.1. Lemma 6.6 is needed in the proof of uniqueness in the next section.

Lemma 6.1. Let $P^{\Phi_{N}}$ be the law of the process $\xi_{N}(t)=\left(x_{1}(t), \ldots, x_{N}(t)\right)$ with $\Phi_{N}$ as initial distribution and let $P_{N}$ be its law with initial density $a_{N}$ (relative to $\Phi_{N}$ ) such that

$$
\int_{R^{N}} a_{N} \log a_{N} \cdot \Phi_{N} d x \leqq C N
$$

with $C$ a constant. Then

$$
\lim _{l \uparrow^{\infty}} \varlimsup_{N \rightarrow \infty} P_{N}\left\{\sup _{0 \leqq t \leqq 1} \frac{1}{N} \sum_{j=1}^{N}\left|x_{j}(t)\right| \geqq l\right\}=0 .
$$

Proof. By Lemma 1.12 of $[\mathrm{K}-\mathrm{V}]$ we have that for any symmetric function $g$ on $\mathbb{R}^{N}$

$$
P^{\Phi_{N}}\left\{\sup _{0 \leqq t \leqq 1} g\left(\xi_{N}(t)\right) \geqq l\right\} \leqq \frac{3}{l} \sqrt{A+B},
$$


where

$$
\begin{gathered}
A=\int_{R^{N}} g^{2} \Phi_{N} d x \\
B=\frac{N^{2}}{2} \int_{R^{N}}\left\{\sum_{i=0}^{N-1} \frac{1}{g}\left(\frac{\partial g}{\partial x_{i}}-\frac{\partial g}{\partial x_{i+1}}\right)^{2}\right\} \Phi_{N} d x
\end{gathered}
$$

are assumed finite. Let

$$
g=\exp \left\{\sum_{i=0}^{N-1}\left|x_{i}\right|\right\} .
$$

Then there are constants $C_{1}$ and $C_{2}$ independent of $N$ such that

Therefore

$$
A \leqq C_{1}^{N} \text { and } B \leqq N^{2} C_{2} C_{1}^{N} .
$$

$$
P^{\Phi_{N}}\left\{\sup _{0 \leqq t \leqq 1} \frac{1}{N} \sum_{i=0}^{N-1}\left|x_{i}(t)\right| \geqq l\right\} \leqq \frac{3}{e^{N l}}\left(C_{1}^{N}+N^{2} C_{2} C_{1}^{N}\right),
$$

which is equivalent to

$$
P^{\Phi_{N}}\left\{\sup _{0 \leqq t \leqq 1} \frac{1}{N} \sum_{i=0}^{N-1}\left|x_{i}(t)\right| \geqq l\right\} \leqq C_{3} e^{-C_{4} N l}
$$

for some constants $C_{3}$ and $C_{4}$.

We now use the basic inequality (2.3) which is

$$
\int g f_{N} \Phi_{N} d x \leqq \log \int e^{g} \Phi_{N} d x+\int f_{N} \log f_{N} \cdot \Phi_{N} d x,
$$

to obtain the inequality

$$
P_{N}(A) \leqq \frac{\log 2+H_{N}}{\log \left(1+1 / P^{\Phi_{N}}(A)\right)}
$$

for any Borel set $A$ in $\mathbb{R}^{N}$. Here $H_{N}$ is the entropy of the law $P_{N}$ relative to $P^{\Phi_{N}}$ and we know by hypothesis that $H_{N} \leqq C N$. This inequality and the estimate above give

$$
P_{N}\left\{\sup _{0 \leqq t \leqq 1} \frac{1}{N} \sum_{i=0}^{N-1}\left|x_{i}(t)\right| \geqq l\right\} \leqq \frac{\log 2+C N}{\log \left(1+1 / C_{3} e^{-C_{4} N l}\right)} .
$$

This implies that

$$
\varlimsup_{N \rightarrow \infty} P_{N}\left\{\sup _{0 \leqq t \leqq 1} \frac{1}{N} \sum_{i=1}^{N-1}\left|x_{i}(t)\right| \geqq l\right\} \leqq \frac{C_{5}}{l}
$$

for some constant $C_{5}$ and hence the lemma is proved.

Lemma 6.2. For every test function $J$ on $S$,

$$
\lim _{\delta \downarrow 0} \varlimsup_{N \rightarrow \infty} P_{N}\left\{\sup _{\substack{0 \leqq t, s \leq 1 \\|--s| \leqq \delta}}\left|\left\langle J, \mu_{N}(t)\right\rangle-\left\langle J, \mu_{N}(s)\right\rangle\right|>\varepsilon\right\}=0
$$

for all $\varepsilon>0$.

Proof. By Itô's formula and a calculation similar to the one in Sect. 5 for the 
identification we see that we must show that

$$
\varlimsup_{N \rightarrow \infty} P_{N}\left\{\sup _{\substack{0 \leqq s, t \leq 1 \\|s-t| \leqq \delta}}\left|\int_{s}^{t} \frac{1}{N} \sum_{j=1}^{N} J^{\prime \prime}\left(\frac{j}{N}\right) \phi^{\prime}\left(x_{j}(\sigma)\right) d \sigma\right|>\varepsilon\right\} \rightarrow 0
$$

as $\delta \rightarrow 0$ for all $\varepsilon>0$. In fact since $\left|J^{\prime \prime}\right|$ is bounded it suffices to show that

$$
\lim _{\delta \downarrow 0} \varlimsup_{N \rightarrow \infty} P_{N}\left\{\sup _{\substack{0 \leqq \leqq, t \leqq 1 \\|s-t| \leqq \delta}}\left|\int_{s}^{t} \frac{1}{N} \sum_{j=1}^{N} \phi^{\prime}\left(x_{j}(\sigma)\right) d \sigma\right|>\varepsilon\right\}=0
$$

To prove this we use a truncation $\psi_{l}$ of $\phi^{\prime}$ defined by

$$
\phi^{\prime} \text { for }\left|\phi^{\prime}\right| \leqq l, \quad \psi_{l}=l \text { for } \phi^{\prime}>l, \quad-l \text { for } \phi^{\prime}<-l \text {. }
$$

It then suffices to show that the estimate is valid for $\phi^{\prime}$ replaced by $\psi_{l}$, which is obvious since $\psi_{l}$ is bounded, and then that

$$
\lim _{l \uparrow \infty} \varlimsup_{N \rightarrow \infty} P_{N}\left\{\int_{0}^{1} \frac{1}{N} \sum_{j=1}^{N}\left|\psi_{l}\left(x_{j}(\sigma)\right)-\phi^{\prime}\left(x_{j}(\sigma)\right)\right|>\varepsilon\right\}=0
$$

for all $\varepsilon>0$. Now

$P_{N}\left\{\int_{0}^{1} \frac{1}{N} \sum_{j=1}^{N}\left|\psi_{l}\left(x_{j}(\sigma)\right)-\phi^{\prime}\left(x_{j}(\sigma)\right)\right|>\varepsilon\right\} \leqq \frac{1}{\varepsilon} \int_{0}^{1} E^{P_{N}}\left\{\frac{1}{N} \sum_{j=1}^{N}\left|\psi_{l}\left(x_{j}(\sigma)\right)-\phi^{\prime}\left(x_{j}(\sigma)\right)\right|\right\} d \sigma$,

and for the expectation on the right we can use the basic inequality (2.3). This gives for any $0 \leqq t \leqq 1$ and any $\gamma>0$,

$$
\begin{aligned}
\int_{R^{N}} \frac{1}{N} \sum_{j=1}^{N}\left|\psi_{l}\left(x_{j}\right)-\phi^{\prime}\left(x_{j}\right)\right| f_{N} \Phi_{N} d x & \leqq \frac{1}{N \gamma} \log \left(\int_{R} e^{\gamma\left|\psi_{l}-\phi^{\prime}\right|-\phi} d x\right)^{N}+\frac{1}{N \gamma} \int f_{N} \log f_{N} D_{N} d x \\
& \leqq \frac{c}{\gamma}+\frac{1}{\gamma} \log \left(\int_{\left|\phi^{\prime}\right|>l} e^{\gamma\left|\phi^{\prime}\right|-\phi} d x\right) .
\end{aligned}
$$

Thus

$$
\lim _{l \rightarrow \infty} \varlimsup_{N \rightarrow \infty} P_{N}\left\{\int_{0}^{1} \frac{1}{N} \sum_{j=1}^{N}\left|\psi_{l}\left(x_{j}(\sigma)\right)-\phi^{\prime}\left(x_{j}(\sigma)\right)\right| d \sigma>\varepsilon\right\} \leqq \frac{c}{\gamma},
$$

where we use the hypothesis that

$$
\int e^{\left.\gamma \mid \phi^{\prime}\right\}-\phi} d x<\infty
$$

for all $\gamma>0$. Letting $\gamma \rightarrow \infty$ gives the result.

Lemma 6.3. Let $f_{N}$ be the density relative to $\Phi_{N}$ of a random variable $\xi_{N}=$ $\left(x_{1}, x_{2}, \ldots, x_{N}\right)$ on $\mathbb{R}^{N}$ such that

$$
\int_{R^{N}} f_{N} \log f_{N} \Phi_{N} d x \leqq C N
$$

with $C$ independent of $N$. Let $Q_{N}$ be the law of the empirical measure

$$
\mu=\frac{1}{N} \sum_{j=1}^{N} \delta_{j / N} x_{j} .
$$


Then for any weak limit $Q$ of $Q_{N}$

$$
Q\{\mu: \mu \ll d \theta\}=1,
$$

and if $m(\theta)=d \mu / d \theta$ then

$$
E^{Q}\left\{\int_{S} h(m(\theta)) d \theta\right\} \leqq C,
$$

where $C$ is the constant in $(6.1)$ and $h(x)$ is the conjugate convex function of $\rho(\lambda)=\log M(\lambda), M(\lambda)=\int e^{\lambda y-\phi(y)} d y$.

Proof. Let $W^{N}$ be the law of $\mu$ induced by $\Phi_{N}$ itself. Under $W^{N}, \mu$ is the empirical measure of $N$ independent, identically distributed random variables with mean

$$
m=\int x e^{-\phi(x)} d x,
$$

and finite moments of all orders. The law of large numbers tells us that $W^{N}$ converges weakly to the deterministic law concentrated on the measure $m d \theta$ (Lebesgue measure multiplied by $m$ ). Moreover $W^{N}$ has the large deviations property [V] with I function,

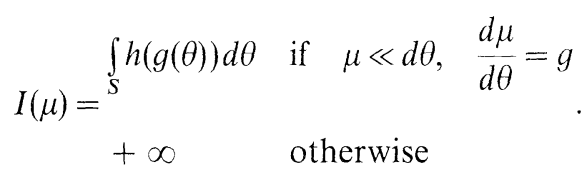

The large deviations property leads also to the result

$$
\lim _{N \rightarrow \infty} \frac{1}{N} \log \int e^{N u} \Phi_{N}=\sup \left\{u(\mu)-\int_{S} h(g(\theta)) d \theta\right\}
$$

for any bounded, continuous function $u$ of $\mu$.

Let $\left\{f_{j}\right\}, j=1,2, \ldots$ be a sequence of functions on $S$ that are dense in $C(S)$ and let

$$
u_{k}(\mu)=\sup _{1 \leqq J \leqq k}\left\{\int_{S} f_{j}(x) \mu(d \theta)-\int_{S} \rho\left(f_{j}(\theta)\right) d \theta\right\} .
$$

We can pass to the limit in the basic inequality (2.3) in the form

$$
\int_{R^{N}} u_{k} f_{N} \Phi_{N} d x \leqq \frac{1}{N} \log \int e^{N u_{k}} \Phi_{N} d x+\frac{1}{N} \int f_{N} \log f_{N} \cdot \Phi_{N} d x .
$$

Since the entropy per variable is bounded by $C$ we conclude that

$$
E^{Q}\left\{u_{k}(\cdot)\right\} \leqq \sup _{\left\{\mu \cdot d \mu / d \theta=g \in L_{1}\right\}}\left\{u_{k}(\mu)-\int_{S} h(g(\theta)) d \theta\right\}+C .
$$

By convex duality

$$
I(M)=\int_{S} h(g(\theta)) d \theta=\sup _{f \in C(S)}\left\{\int_{S} f(\theta) \mu(d \theta)-\int_{S} \rho(f(\theta)) d \theta\right\} \geqq u_{k}(\mu) .
$$

Using this we get the estimate

$$
E^{Q}\left\{u_{k}(\cdot)\right\} \leqq C
$$


Since $u_{k}(\mu) \rightarrow I(\mu)$ as $k \rightarrow \propto$, by Fatou's lemma

This implies that

$$
E^{Q}\{I(\cdot)\} \leqq C .
$$

and that

$$
Q\{\mu: \mu \ll d \theta\}=1
$$

where $d \mu / d \theta=m(\theta)$.

$$
E^{Q}\left\{\int_{S} h(m(\theta)) d \theta\right\} \leqq C
$$

Lemma 6.4. Let $\psi_{l}(x)$ be a truncation of $\phi^{\prime}(x)$.

$$
\begin{aligned}
& \phi^{\prime}(x),\left|\phi^{\prime}(x)\right| \leqq l \\
& \psi_{l}(x)=l \quad, \quad \phi^{\prime}(x)>l \\
&-l, \quad \phi^{\prime}(x)<-l
\end{aligned} .
$$

Let

$$
\tilde{\psi}_{l}(x)=\frac{1}{M(\lambda)} \int e^{\langle y-\phi(y)} \psi_{l}(y) d y
$$

with $\lambda=h^{\prime}(x)$. Then for each $x$,

$$
\widetilde{\psi}_{l}(x) \rightarrow h^{\prime}(x) \text { as } l \rightarrow \infty .
$$

Proof. We will use our hypotheses

Clearly

$$
\begin{array}{ll}
\int e^{\prime x-\phi(x)} d x<\infty & \text { for all } \lambda, \\
\int e^{\sigma\left|\phi^{\prime}(x)\right|-\phi(x)} d x<\infty & \text { for all } \sigma .
\end{array}
$$

$$
\int e^{\sigma\left|\psi_{1}-\phi^{\prime}\right|-\phi} d x \rightarrow 1 \quad \text { on } l \rightarrow \infty,
$$

for all $\sigma \geqq 0$. Now

$$
\frac{1}{M(\lambda)} \int e^{\lambda y-\phi(y)} \phi^{\prime}(y) d y=\lambda=h^{\prime}(x) .
$$

Hence

$$
\left|\widetilde{\psi}_{l}(x)-h^{\prime}(x)\right|=\left|\frac{1}{M(\lambda)} \int e^{\lambda y-\phi(y)}\left(\psi_{l}(y)-\phi^{\prime}(y)\right) d y\right| \leqq \int \frac{e^{\lambda y-\phi(y)}}{M(\lambda)}\left|\psi_{l}-\phi^{\prime}\right| d y .
$$

By the basic inequality (2.3), for any $\sigma>0$,

$$
\begin{aligned}
\int \frac{e^{\prime y-\phi(1)}}{M(\lambda)}\left|\psi_{l}-\phi^{\prime}\right| d y & \leqq \frac{1}{\sigma} \log \int e^{\sigma\left|\psi_{l}-\phi^{\prime}\right|-\phi} d y+\frac{1}{\sigma} \int \frac{e^{\lambda y}}{M(\lambda)}[\lambda y-\rho(\lambda)] e^{--\phi(y)} d y \\
& =\frac{1}{\sigma} \log \int e^{\sigma\left|\psi_{l}-\phi^{\prime}\right|-\phi} d y+\frac{h(x)}{\sigma}
\end{aligned}
$$

Here we use the fact that at $\lambda=h^{\prime}(x)$,

$$
\frac{1}{M(\lambda)} \int y e^{\prime y-\phi(v)} d y=x
$$

Passing to the limit for $x$ fixed we see that 


$$
\varlimsup_{l \rightarrow \infty}\left|\tilde{\psi}_{l}(x)-h^{\prime}(x)\right| \leqq \frac{h(x)}{\sigma}
$$

because of our basic hypotheses on $\phi$ and $\phi^{\prime}$. Letting now $\sigma \rightarrow \infty$ we obtain the desired result.

Lemma 6.5. There is a constant $C_{\varepsilon}$ such that for any $\varepsilon>0$,

$$
\left|h^{\prime}(x)\right| \leqq C_{\varepsilon}+\varepsilon h(x) .
$$

Proof. We will actually show that

$$
\varlimsup_{l \rightarrow \infty}\left|\tilde{\psi}_{l}(x)\right| \leqq C_{\varepsilon}+\varepsilon h(x),
$$

which combined with Lemma 6.4 gives the result.

Now

$$
\widetilde{\psi}_{l}(x)=\frac{1}{M(\lambda)} \int e^{\lambda y-\phi(y)} \psi_{l}(y) d y
$$

and as in the proof of Lemma 6.4

$$
\left|\tilde{\psi}_{l}(x)\right| \leqq \frac{1}{\sigma} \log \int e^{\sigma\left|\psi_{l}(v)\right|-\phi(v)} d y+\frac{h(x)}{\sigma}
$$

for any $\sigma>0$. With

we then get

$$
\tilde{C}_{\sigma}=\log \int e^{\sigma\left|\phi^{\prime}\right|-\phi} d y
$$

$$
\varlimsup_{l \rightarrow \infty}\left|\tilde{\psi}_{l}(x)\right| \leqq \frac{\tilde{C}_{\sigma}}{\sigma}+\frac{1}{\sigma} h(x) .
$$

If we let $\varepsilon=1 / \sigma$ and $C_{\varepsilon}=\varepsilon \widetilde{C}_{1 / \varepsilon}$ this is the same as the statement above.

Lemma 6.6. Let us assume that the conditions of Lemma 6.3 are fulfilled and in addition

$$
\frac{1}{8} \int_{S} \frac{1}{f_{N}} \sum_{i=1}^{N}\left(\frac{\partial f_{N}}{\partial x_{i}}-\frac{\partial f_{N}}{\partial x_{i+1}}\right)^{2} \Phi_{N} d x \leqq \frac{C^{\prime}}{N},
$$

where $C^{\prime}$ is some nonnegative constant. Then any limit point $Q$ obtained in that lemma satisfies in addition

$$
E^{Q}\left[\int_{S}\left[\frac{\partial}{\partial \theta} h^{\prime}(m(\theta))\right]^{2} d \theta\right] \leqq 8 C^{\prime}
$$

with the same constant $C^{\prime}$ as in (6.2).

Proof. This follows immediately from Eq. (4.22).

\section{Uniqueness}

We will show in this section that a weak solution of the equation

$$
\frac{\partial u}{\partial t}=\frac{1}{2}\left(h^{\prime}(u)\right)_{\theta \theta}, \quad u(0, \theta)=f(\theta)
$$


is unique within the class of measures $\mu(t, d \theta)$ satisfying

$$
\sup _{0 \leqq t \leqq T}\|\mu(t, \cdot)\|<\infty \text { and } \mu(t, \cdot) \text { is weakly continuous in } t
$$

$\mu(t, d \theta)=u(t, \theta) d \theta$ for almost all $t$ and

$$
\begin{gathered}
\int_{0}^{T} \int_{S} h(u(t, \theta)) d \theta<\infty, \\
\int_{0}^{T} \int_{S}\left\{\left[h^{\prime}(u(t, \theta))\right]_{\theta}\right\}^{2} \leqq C<\infty .
\end{gathered}
$$

A weak solution is of course given by

$$
\int_{S} J(\theta) \mu(t, d \theta)-\int_{S} J(\theta) \mu(0, d \theta)=\int_{0}^{t} \int_{S} \frac{1}{2} J^{\prime \prime}(\theta) h^{\prime}(u(s, \theta)) d s d \theta,
$$

for all smooth test functions $J(\cdot)$.

We first take $J=1$ and check that $\int u(t, \theta) d \theta=\int f(\theta) d \theta=a$ for all $t$ and all solutions. The function $u(t, \theta)-a$ is denoted by $\tilde{u}(t, \theta)$ and has mean zero on $S$. Therefore there is a function $\tilde{v}(t, \theta)$ of mean zero, such that

$$
\frac{\partial \tilde{v}(t, \theta)}{\partial \theta}=\tilde{u}(t, \theta)
$$

and (7.5) can be rewritten in a suitable sense as

$$
\frac{\partial}{\partial t} \int_{S} \tilde{u}(t, \theta) J(\theta) d \theta=\frac{1}{2} \int_{S} J^{\prime \prime}(\theta)\left[h^{\prime}(u(t, \theta))\right] d \theta
$$

or

$$
\frac{\partial}{\partial t} \int_{S} J^{\prime}(\theta) \tilde{v}(t, \theta) d \theta=\frac{1}{2} \int_{S} J^{\prime}(\theta)\left[h^{\prime}(u(t, \theta))\right]_{\theta} d \theta .
$$

Because of (7.4) this means that $\tilde{v}(t, \cdot)$ is differentiable in $t$ as a map into $L_{2}(S)$ and

$$
\frac{d \tilde{v}}{d t}=\frac{1}{2}\left[h^{\prime}(u(t, \theta))\right]_{\theta} .
$$

Now if we denote the difference between two solutions by $w$, then $w=u-v=\tilde{u}-\tilde{v}$ and

$$
\frac{d w}{d t}=\frac{1}{2}\left[h^{\prime}(u(t, \theta))\right]_{\theta}-\frac{1}{2}\left[h^{\prime}(v(t, \theta))\right]_{\theta}
$$

If we now calculate

$$
\begin{aligned}
\frac{d}{d t}\|w\|^{2} & =\int_{S} w(t, \theta)\left[h^{\prime}(u(t, \theta))-h^{\prime}(v(t, \theta))\right]_{\theta} d \theta \\
& =-\int_{S}\left(h^{\prime}(u(t, \theta))-h^{\prime}(v(t, \theta))\right)(u(t, \theta)-v(t, \theta)) d \theta \\
& \leqq 0 .
\end{aligned}
$$


Since $\|w(0)\|=0$ we are done. The steps need a little bit of justification but it is routine.

\section{Concluding Remarks}

There have been several examples where hydrodynamic limits have been established for interacting systems. For example De Masi et al. [DE] and Dobrusin [D]. See also Spohn [S] for the closely related problem of fluctuation theory. Our work is related to and was motivated by the work of Fritz [FR]. Funaki [FU] has some results extending Fritz's work. But our methods are very different from all of the earlier methods. We use basically entropy estimates. They have the advantage of universality and with modifications the method should be applicable to other models that are reversible and when the scaling is of diffusion type. Moreover the method allows us to proceed farther and do large deviation theory. See for instance Donsker and Varadhan [DV]. Although we treat the case of dimension one it is not an essential condition. With only a change in notation the method works in any number of dimensions.

Replacing the circle or torus by $R^{d}$ poses more of a challenge. The problem in some sense is essentially local, and it should be possible to localize. Fritz has conveyed to us some ideas in private communication, but it still remains to be done.

\section{References}

[KV] Kipnis, C., Varadhan, S. R. S.: Central limit theorem for additive functionals of reversible Markov processes and applications to simple exclusions. Commun. Math. Phys. 104, 1-19 (1986)

[DE] De Masi, A., Ianiro, N., Pellegrinotti, A., Presutti, E.: A survey of the hydrodynamical behavior of many-particle systems. In: Nonequilibrium phenomena II. From stochastics to hydrodynamics. Lebowitz, J. L., Montroll, E. N. (eds.) pp. 123-294. Amsterdam: North-Holland 1984

[D] Dobrushin, R., Seigmund-Schultze, R.: The hydrodynamic limit for systems of particles with independent evolution, Akademie der Wissenschaften der DDR, Berlin, 1981

[S] Spohn, H.: Equilibrium fluctuations for interacting Brownian particles. Commun. Math. Phys. 103, 1-33 (1986)

[FR] Fritz, J.: On the hydrodynamic limit of a scalar Ginzburg-Landau lattice model, IMA Vol. 9. Berlin, Heidelberg, New York: Springer 1987

[F] Funaki, T.: preprint 1987

[DV] Donsker, M., Varadhan, S. R. S.: preprint 1987

[V] Varadhan, S. R. S.: Asymptotic probabilities and differential equations. Commun. Pure Appl. Math. 19, 261-286 (1966)

Communicated by J. L. Lebowitz

Received October 29, 1987 
